\title{
Robust characterization of the vibrational behaviour of light assembled structures with random parameters
}

\author{
Martin GHIENNE ${ }^{a, b}$ \\ Luc LAURENT ${ }^{b}$ \\ Claude BLANZÉ ${ }^{b}$ \\ ${ }^{a}$ Laboratoire Quartz EA7393, Institut Supérieur de Mécanique de Paris - SUPMECA, \\ 3 rue Fernand Hainaut, 93407 Saint-Ouen Cedex, France \\ Tel.: +33-1-49-45-29-67 \\ martin.ghienne@supmeca.fr \\ ${ }^{b}$ Laboratoire de Mécanique des Structures et des Systèmes Couplés, Conservatoire \\ national des arts et métiers, case courrier 2D6R10, 2 rue Conté, 75003 Paris, France \\ Tel.: +33-1-58-80-85-80 \\ luc.laurent@lecnam.net \& claude.blanze@lecnam.net
}

\begin{abstract}
Predictive simulations of assembled structures raises some computational challenges for usual industrial structures. It is indeed necessary to deal with non-linear phenomena, such as contact or friction, and to consider sources of uncertainties mainly responsible for the deviation between the effective behaviour of the structure and results from deterministic simulations. Each of these two features of assembled structures requires intensive computational resources and the significant number of joints of actual structures increases even more the computational cost. In order to address such problems, this work aims to provide a pragmatic approach to robustly characterize the vibrational behaviour of light assembled structures considering the variability of the parameters of the joints. All the uncertainties of the whole problem are assumed to be associated to the joint interfaces. First, a nominal model of a joint consistent with the studied structure is identified using experimental data. Then a stochastic model of a joint is defined by considering the mechanical design variables of the nominal model as random variables. Based on an approximation of the maximum likelihood principle, the parameters of this stochastic model of joint are identified. A straightforward non-intrusive strategy referred to as the Stochastic Model Reduction (SMR) approach is used in the identification procedure of the stochastic model of the joint. The ease of implementation and the efficiency of the SMR approach justify this choice. The proposed approach is applied to an academic structure which is representative of light assembled structures: a three beam bolted frame. A stochastic model of joint characterized by its stiffness is thus implemented for each elementary joint of this frame. It allows us to characterize the variability of the vibrational behaviour of the frame taking into account the variability of each joint. Finally the comparison of the predicted stochastic response with the experimental behaviour of the structure shows that the proposed approach allows to tackle the variability of the actual structure. The proposed approach has been developed in the purpose of industrial applications. Based on a pragmatic modeling of the joint, a non-intrusive numerical tool and a stochastic modeling, the whole strategy could be indeed easily implemented on more complex industrial assemblies.
\end{abstract}

Keywords: Robust design - parametric uncertainty - bolted joints - stochastic joint model stochastic finite element method - stochastic model reduction - random stiffness identification

- Open Archive HAL with file: hal-02387438

- Doi: 10.1016/j.ymssp. 2019.106510 


\section{Contents}

1 Introduction

2 A nominal model of joint based on experimental macroscopic observation 5

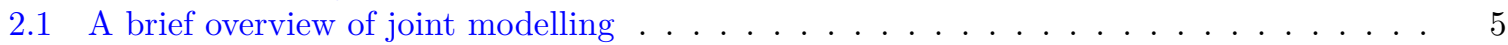

2.2 Experimental identification of the joint behaviour . . . . . . . . . . . . 7

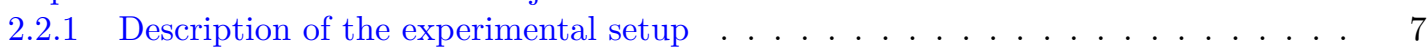

2.2 .2 Identification of the joint behaviour $\ldots \ldots \ldots \ldots \ldots$

3 Uncertainties for assemblies on the context of vibration 12

3.1 State of art . . . . . . . . . . . . . . . . . . . . . . 12

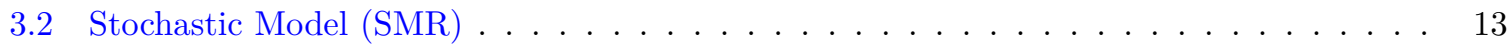

4 Identification of a stochastic model of joint 15

4.1 Identification process with uncertainties . . . . . . . . . . . . . . . 15

4.2 Identification of the stochastic model of joint $\ldots \ldots \ldots \ldots \ldots \ldots$

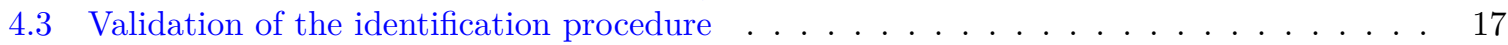

5 Robust characterization of the vibrational behaviour of multi-bolted frame 19

5.1 Two-bolted frame . . . . . . . . . . . . . . . . . . . . . . . . . . . 19

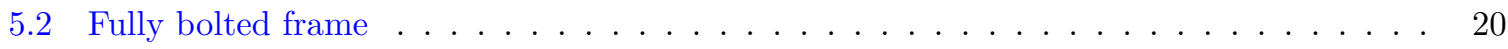

6 Conclusion 22

$\begin{array}{ll}\text { References } & 23\end{array}$

\section{Introduction}

Taking into consideration the variability of parameters during the design phase of a structure allows especially to design solutions more robust to deviations between the actual system and the nominal designed system. This design approach can be defined as robust design [1] as proposed by Zang, Friswell, and Mottershead [2]: "A product or process is said to be robust when it is insensitive to the effects of sources of variability, even through the sources themselves have not been eliminated". In other terms, the design of the system is then achieved in order to be independent of the variability of the actual system. Focusing on the vibrational behaviour of a structure, this would allow to robustly design devices dedicated to vibration reduction which could be mistuned if the effective vibrational behaviour of the structure differs from the expected one simulated during the design phase. For instance piezoelectric devices [3-5] or tuned mass dampers [6, 7] could be considered for such purpose. The formulation of an efficient and accurate stochastic model paves the way for the use of advanced design methods based for example on robust optimisation [8] or reliability-based optimisation [9].

Different sources of uncertainties can lead to such variability. Uncertainties are referred to as parametrical uncertainties when they concern variability in design parameters and to as non-parametrical uncertainties when they are associated to a lack of knowledge on the considered model. Relying on the probability theory, the probabilistic approach of uncertainties provides a powerful, efficient and effective tool for modeling and for solving direct and inverse problems [10,11]. The developments presented in this paper are addressed in this probabilistic framework.

This work focuses on light assembled structures which include localized sources of uncertainties, such as friction and contact in the joint interfaces, boundary conditions or defective parts. We consider the simplified case where the assembled structure could be modelled considering a separation of difficulties [12]: the parts of the assembly are assumed to be deterministic and with a linear mechanical behavior whereas the interfaces between parts incorporate all the uncertainties of the whole problem and could be weakly non-linear. These uncertainties are assumed to be mainly responsible for deviation between the 

of light assembled structures with random parameters. Mechanical Systems and Signal Processing, 136, 2020. doi: 10.1016/j.ymssp.2019.106510

effective behaviour of the structure and results from deterministic simulations for that kind of structures. Light assembled structures can be defined as a significant number of slender sub-structures having an elastic linear behaviour assembled together with joints. Joints involve non-linear phenomena as contact and friction in their interface, affecting the vibrational behaviour of the overall assembled structure. Taking into account these phenomena in a numerical model to simulate the behaviour of a single joint could be time consuming and, therefore it would be difficult to consider an entire lightweight structures assembled with numerous joints. In addition considering uncertainties with classical methods also requires intensive computations. The robust characterization of the vibrational behaviour of industrial assembled structures using direct methods is therefore difficult to achieve. The main purpose of this work is to provide a straightforward framework for the robust design of light assembled structures. Considering light structures enables us to ensure a well-known behaviour. Extension on massive 3D assembled structures (such as $[13,14]$ ) could be easily considered but will not be addressed in this paper.

The literature reviews numerous models allowing to take into account the influence of the joints on assembled structures with different modelling scales. Three scales of description could be identified to describe the behaviour of a joint: the smallest scale considers the local behaviour of the joint interface, the intermediate scale aims to describe a global function between loads applied on each assembled part and displacements in the interface, the broader scale aims to describe the influence of the joint on the behaviour of the overall assembled structure without describing the effective local displacements of the assembled parts in the vicinity of the joint interface. This classification is proposed in the scope of this work and aims to distinguish a scale where each part of the joint is considered (meso scale) and an overall scale (macro scale) "from the structure point of view" where the joint can be seen as a part of the structure with specific dynamical properties. The underlying idea is that, from the local contact description of the macro scale point of view, these models require more or less computing resources depending on their ability to describe the joint physics. This classification is briefly developed in the next Section. The most suitable model to characterize the vibrational behaviour of light assembled structures with a significant number of joints is then identified.

Even if numerous models of joint can be found in the literature, modelling joints and simulating assembled structures considering uncertainties is not so widespread. Ghanem et al. [15] consider the case of a space-frame constituted of homogeneous deterministic segments connected to each other by a band of material whose Young's modulus is modelled as a spatially varying stochastic process. The stochastic vibrational problem is solved using a spectral method. Even if the studied structure could be assimilated to a bolted frame, the purpose of this work is not dedicated to the vibrational characterization of assembled structures and no particular conclusion could be drawn on this topic. Mignolet, Song, and Wang [16] consider a Monte-Carlo simulation to compare the variability of the dynamical properties of a joint to experimental data. A stochastic model of joint is proposed based on the Iwan model $[17,18]$ and identified using a maximum likelihood strategy. This approach requires experimental data obtained on a single joint isolated from the global assembled structure, which is not always possible. Daouk et al. [19] study the dynamical behaviour of an assembled structure considering uncertainties in the joint stiffness of an industrial structure. They compare three classical parametric probabilistic methods referred to as the Monte-Carlo Simulation [20], the Perturbation method [21] and the Subspace Reduction method [22], to a non probabilistic approach referred to as the Lake Of Knowledge (LOK) theory [23]. The LOK theory is an uncertainty propagation technique suitable to epistemic uncertainty characterization which may also take into account random uncertainties. Non probalistic interval's approaches could be also considered to study the influence of the uncertainties of parameters on the natural frequencies and on frequency response functions [24-26]. The present work assumes that the variability of the joints dynamical properties is mainly due to the system parameter's randomness. A probabilistic framework is thus preferred hereafter.

The aim of this work is to provide numerical tools for the robust design of light assembled structures. Considering a given assembled structure, the proposed approach is to first identify a "just sufficient" nominal model of the studied joints. By "just sufficient" we mean a model with a minimum number of parameters (here the stiffnesses of the joints), a low computational cost and just sufficient to reflect the observable physics. This approach aims to be driven by the actual behaviour of an assembled structure. 
The nominal model of the studied joints will thus be settled based on experimental observations. In a second phase, a stochastic model of joint is defined by randomizing the parameters of the nominal model. Randomizing means here to define a probability density function that characterizes the stochastic behaviour of the mechanical design variables ${ }^{1}$ using the maximum entropy principle. Finally, the characteristics of the parameters of this stochastic model of joint ${ }^{2}$ are identified using an approximation of the maximum likelihood principle. A straightforward non-intrusive approach developed in previous work $[27,28]$ and referred to as the Stochastic Model Reduction (SMR) approach is used to simulate the vibrational behaviour of the structure. The efficiency of this approach allows to integrate it in the identification procedure of a stochastic model of joint. To facilitate the understanding, the proposed approach will be applied to an academic structure representative of light assembled structures: a bolted frame inspired by the work of Van Buren et al. [29]. An illustration of this frame is presented on Figure 1.

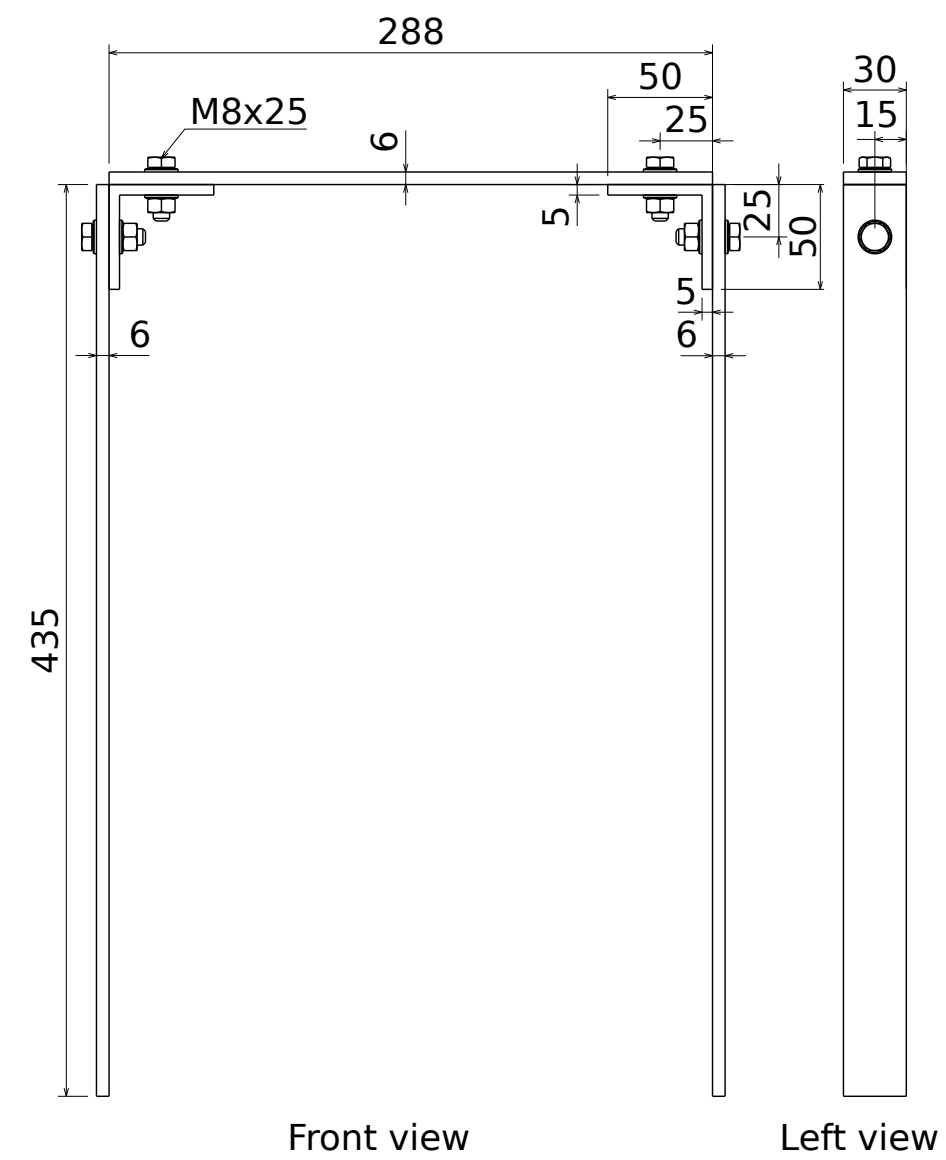

Figure 1: Geometrical characteristics of the studied frame structure (dimensions in $\mathrm{mm}$, beams and angles brackets material: standard aluminium, bolts: M $8 \times 25$ - class 8.8$)$.

The first part of this paper aims to pick a nominal model of the studied joint. After reviewing classical models of joints, a model adapted to the studied structure is selected and identified according to experimental observations. An effective numerical framework for the characterization of the vibrational behaviour of structures with random parameters is then developed. Based on this numerical tool, the

\footnotetext{
${ }^{1}$ The terms "design variables" is here used to designate the mechanical parameters in opposition of the parameters which governed the probability distributions.

${ }^{2}$ In other words the parameters of the probability density functions associated to the mechanical design variables defined by the nominal model of the joint.
} 
Martin Ghienne, Luc Laurent \& Claude Blanzé. Robust characterization of the vibrational behaviour of light assembled structures with random parameters. Mechanical Systems and Signal Processing, 136, 2020. doi: 10.1016/j.ymssp.2019.106510

\begin{tabular}{|c|c|c|c|}
\hline Scale & Macroscopic & Mesoscopic & Microscopic \\
\hline Details & $\begin{array}{l}\text { - dedicated finite ele- } \\
\text { ments } \\
\text { - zero-thickness } \\
\text { element } \\
- \text { thin layer ele- } \\
\text { ment } \\
- \text { node-to-node } \\
\text { element } \\
- \text {... } \\
\text { - dedicated stiffness } \\
\text { applied on nodes } \\
\text { corresponding to } \\
\text { the joint area }\end{array}$ & $\begin{array}{l}\text { - dedicated be- } \\
\text { haviour (elasto-slip, } \\
\text { Signum-Friction } \\
\text { model...) } \\
\text { - rheological models } \\
\quad-\text { Dahl } \\
\quad-\text { LuGre } \\
- \text { Bouc-Wen } \\
- \text { Iwan } \\
- \text { Valanis } \\
- \text {... }\end{array}$ & $\begin{array}{l}\text { - Hertz theory } \\
\text { - Coulomb's friction } \\
\text { law } \\
\text { - fine description of } \\
\text { the surfaces } \\
\text { - advanced material } \\
\text { behavior on the con- } \\
\text { tact area }\end{array}$ \\
\hline References & {$[29-37]$} & {$[17,18,38-49]$} & {$[50-57]$} \\
\hline
\end{tabular}

Table 1: Summary of the main methods and associated references of the joint modelling.

parameters of a stochastic model of joint are identified using a straightforward procedure. Finally, the robust characterization of the vibrational behaviour of different multi-bolted frames is presented.

\section{A nominal model of joint based on experimental macroscopic observation}

\subsection{A brief overview of joint modelling}

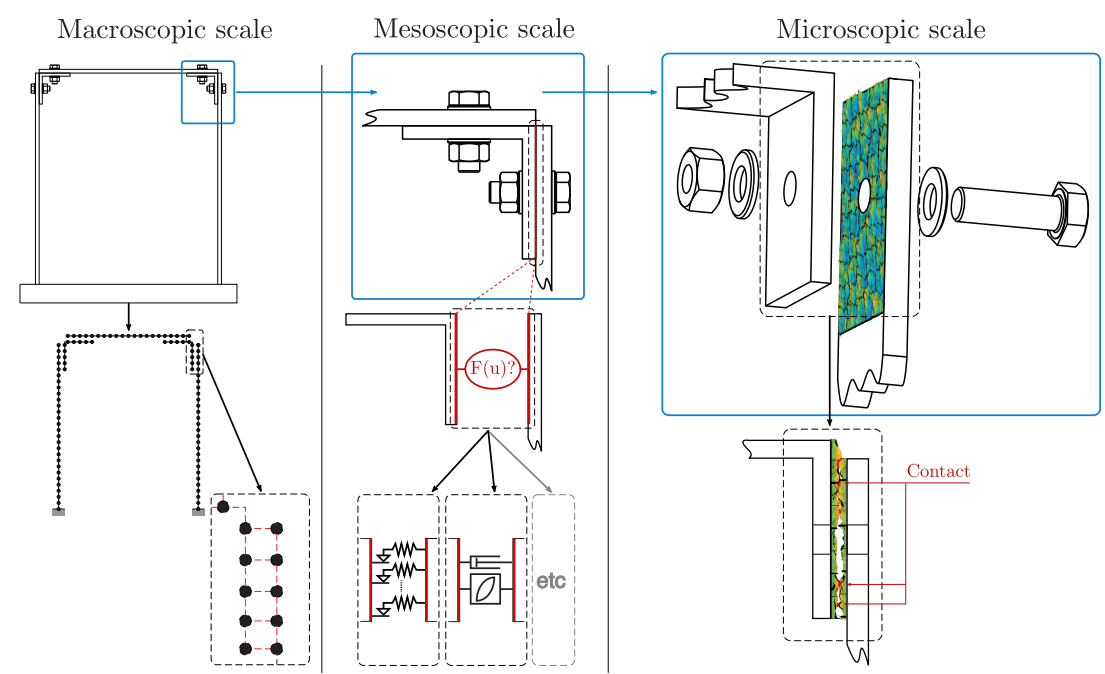

Figure 2: Schematic representation of the scales considered in the joint modelling.

In this Section, a brief overview of joint modelling is presented. Figure 2 and Table 1 summarize the main methods and references presented below. 
Modelling mechanical phenomena in the joint interfaces is defined hereafter as microscopic scale. It is based on constitutive or tribological models providing a local description of the joint. Analytical constitutive models can be found in the literature [50-52] but existing applications are limited to simple geometry of structures with specific loads. Approaches based on finite element discretization of the joint parts allow to tackle more complex geometry [53, 54]. The friction model between elementary contact areas is generally based on Coulomb's law. The main drawback of these approaches is their computational costs: the non-linear problem is mainly solved using iterative algorithms as Newton-Raphson methods and the contact description in the joint interface requires very refined mesh. Tribological models decrease even more the scale of modelling to describe the contact between asperities of each contact surfaces $[55,56]$. They are usually based on Hertz theory and Coulomb's model. These models have a strong dependency to experimental measures and generally assume that the normal preload of the interface is constant over a loading cycle of the joint, which is hardly obtained experimentally. Improved models are also proposed for dealing with specific material behaviour at the contact interface and rough contact surface [57] but these strategies require accurate description of the contact surface. To sum up, constitutive models are not adapted to assembled structures with numerous joints and sub-structures requiring high number of degrees of freedom.

Defined hereafter as mesoscopic scale, the whole joint is modelled using phenomenological or rheological models in order to describe a global function between loads applied on each assembled part and displacements in the interface. They are based on experimental observations and do not necessarily aim to describe the actual physics of the joint interface. Different classical rheological models can be found in the literature [38-41]. The Signum-Friction model is based on Coulomb's law and does not consider sticking of the joint's interface. The Elasto-slip model, also referred to as Jenkins element, Masing element or Coulomb element, takes into account the deformation of the joint prior to interfacial slip. The Dahl model [42] computes the friction force by integrating the joint displacement. The shape of the obtained Force-Displacement hysteresis loop is then tuned using a dedicated parameters. These three models do not consider the Stribeck effect (the friction force first decreases then increases when the friction velocity increases). The Lund Grenoble model [43], (LuGre model), tackles this drawback by considering the joint interface as a set of bristles with a given stiffness and applying a Dahl's model at each bristle end. Initially dedicated to thermoviscoplastic materials, the Valanis model [38, 44] allows to describe macro and micro slips of the joint interface with a single formulation. A non-linear equation involving the relative displacement and velocity of the joint interface and four parameters are used to obtain the friction force. The four parameters have to be identified from a measured hysteresis loop. The Bouc-Wen model $[45,46]$ describes the friction force considering a general hysteresis loop with no direct physical meaning. The main drawback of this model is that it does not satisfy the Masing rule [47, 48] from which, for a loading cycle with total slip of the interface, the instantaneous stiffness at the load reversal point is equal to the sticking stiffness. The last phenomenological model developed here is the Iwan model $[17,18]$. This model allows to describe an hysteresis loop using parallel-serie and serie-parallel networks of Jenkins elements with different sliding thresholds. Using a continuous distribution of Jenkins elements, the number of parameters of this model can be reduced [48, 49]. The four-parameter Iwan model proposed by Segalman [49] is probably one of the most popular one. Phenomenological models presented here describe the behaviour of joint interfaces under shear loads and consider a constant pressure in the joint interface. These models are not adapted if the joint interface is not loaded in shear only, as in the case of a bending moment applied to a lap joint between two beams. To overcome this limitation, Song et al. [30] propose a beam element formulation based on two Iwan models wisely combined.

Finally, the last modelling scale presented here corresponds to the scale of the overall assembled structure. Referred to as macroscopic scale, it aims to characterize the dynamical behaviour of assembled structures with several joints without aiming to describe the actual micro-slip of the joint interfaces. In other terms, only the equivalent stiffness and damping of the joint regarding the overall structure eigenfrequencies and magnitude are considered. Even if approaches as multi-body system approach [31] or spectral element method [32,33] can be found in the literature to model parts of an assembled structure, the most common method uses a finite element discretization of the sub-structures of the assembly. Considering a finite element framework, the influence of joints on the overall assembled 


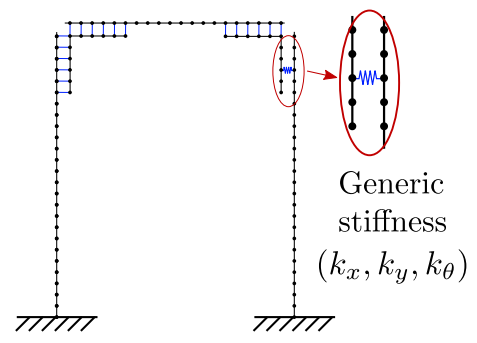

(a) Generic stiffness model.

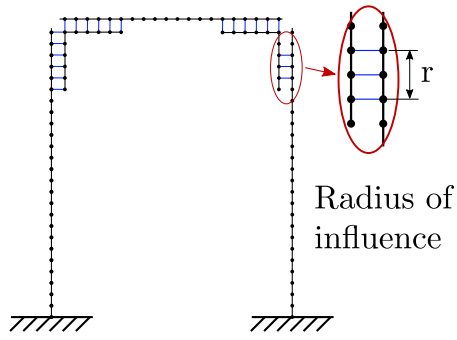

(b) Radius of influence model.

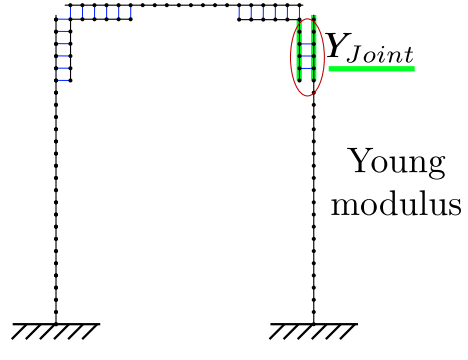

(c) Standard finite element based model.

Figure 3: Various models of macroscopic bolted joint.

structure could be taken into account considering zero-thickness elements [34, 35], thin layer elements [34] or node-to-node elements [29, 30,34,36]. Gant, Champaney, and Rouch [58] proposes a model updating approach considering a simple one-dimensional model of joint and a constitutive relation error. The most straightforward approach consists in modelling the joint with a single node-to-node element. Generic stiffness and damping are then defined between nodes from each assembled part $[29,36]$. An illustration of this model is presented on Figure 3a for the case of the bolted frame considered in this work. This model requires to define stiffness and damping for each degree of freedom at each assembled node. A node-to-node element can also be defined between each node pair of the joint interface. Van Buren et al. [29] used a rigid node-to-node link between node pair in a given radius of influence as shown on Figure 3b. This radius can be seen as a model of the sticking area of the joint interface. A combination between non-rigid elements and radius of influence could also be implemented. Rather than meshing each assembled part with standard elements and adding relations between pair nodes, the last approach consists in meshing all the joints with dedicated elements formulated to take into account the behaviour of the bolted joint as proposed by Ahmadian and Jalali [37] or Song et al. [30]. Ahmadian and Jalali [37] consider a beam finite element formulation to propose a generic definition of the components of the mass and stiffness matrices of a joint. A similar approach is also proposed by Song et al. [30]. This allows, for instance, to implement directly in these matrices the different phenomenological models previously defined. The easiest way to implement this model using a commercial finite element software is to use standard element formulation and to apply dedicated materials properties to the elements corresponding to the joint area. For instance, considering only the stiffness of the joint, an equivalent Young's modulus could be applied to the frame elements corresponding to the joint as illustrated on Figure 3c.

\subsection{Experimental identification of the joint behaviour}

In order to select a model of joint adapted to the studied structure, a preliminary differential analysis has been performed to highlight and quantify the influence of the bolted joint on the behaviour of the assembled frame. The vibrational behaviour of two frames, a frame with only one bolted joint and an equivalent monolithic frame, are thus compared to evaluate the influence of the bolted joint. Only the stiffness of the bolted joint is considered in this paper. The next Section aims to present the experimental setup used in the context of this work.

\subsubsection{Description of the experimental setup}

The experimental setup is constituted of a frame clamped to a base plate. The vibrational behaviour of the frame is characterized using a laser scanning vibrometer and a sweep sine excitation applied using a shaker as illustrated on Figure 4. The considered frequency bandwidth is from 0 to $1250 \mathrm{~Hz}$ chosen to be able to observe the 10 first eigenmodes which remain clearly identifiable during the whole study. The excitation is applied at $11.5 \mathrm{~cm}$ from the frame base, centered on the vertical beam. The frame 


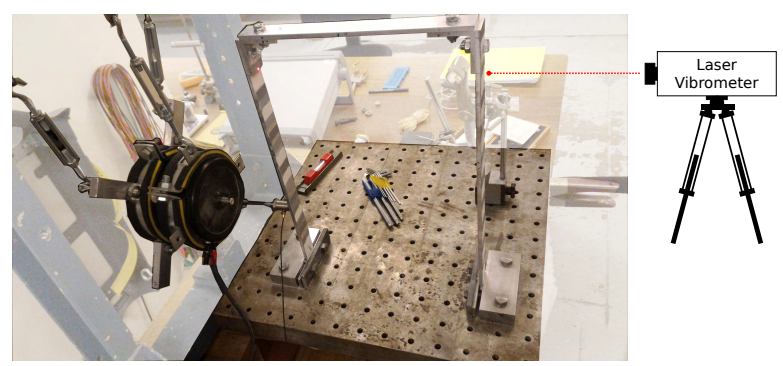

Figure 4: Presentation of the experimental setup used to identified an adapted nominal model of a bolted joint.

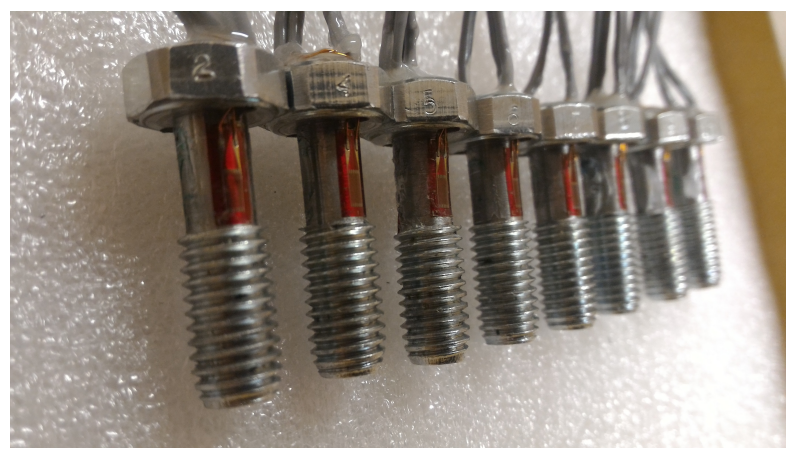

Figure 5: Bolts instrumented with strain gauge.

is assembled with $M 8 \times 25$ steel bolts and adapted steel washers. The Young modulus of the beams constituting the frame has been identified experimentally using a standard tensile-test and is equal to $Y_{\mathrm{alu}}=65.5 \mathrm{GPa}$. The excitation magnitude and the bolt tightening are assumed to be the predominant parameters on the bolted joint behaviour. The experimental setup allows us to study the influence of these two parameters on the joint stiffness.

Each bolt are instrumented with strain gauges, as illustrated on Figure 5, to infer the applied tightening. In the following of this paper, three tightening torques for each bolt referred to as low, medium and high tightening levels will be considered. The preload applied by the joint to the assembled parts is less than $100 \mathrm{~N}$ for the lowest tightening level. It corresponds to a bolt tightened by hand. The medium level corresponds to a preload of $1800 \mathrm{~N}$. The strongest tightening is about $6000 \mathrm{~N}$ and corresponds to the limit of local lamination of the assembled parts. These preload levels will be designated respectively as levels (1), (2) and (3) in the following.

Finally, two magnitudes of excitation are considered. The first, referred to as "low" magnitude is tuned in order to ensure a linear behaviour of the frame. The second, referred to as "high" magnitude, is chosen five times higher. The excitation magnitude is driven by the input signal applied to the shaker.

The clamping system has been designed in order to ensure a repeatable mounting of the frame on the base plate. The vertical beams are positioned in the vertical plane using a plane contact and a three hold point system. The position of the beams is ensured by the clamping pressure applied by a steel vice. The clamped beams are in aluminium, this allows to locally deform the beams to well fit the shape of the vice jaws. The equivalent stiffnesses of the clamping system have been identified in a preliminary experimental study using a model updating technique [28]. It will not be developed here for the sake of brevity.

\subsubsection{Identification of the joint behaviour}

Using the experimental setup previously described, the experimental differential analysis is performed. The Frequency Response Function (FRF) of a monolithic frame and a frame with only one bolted joint 


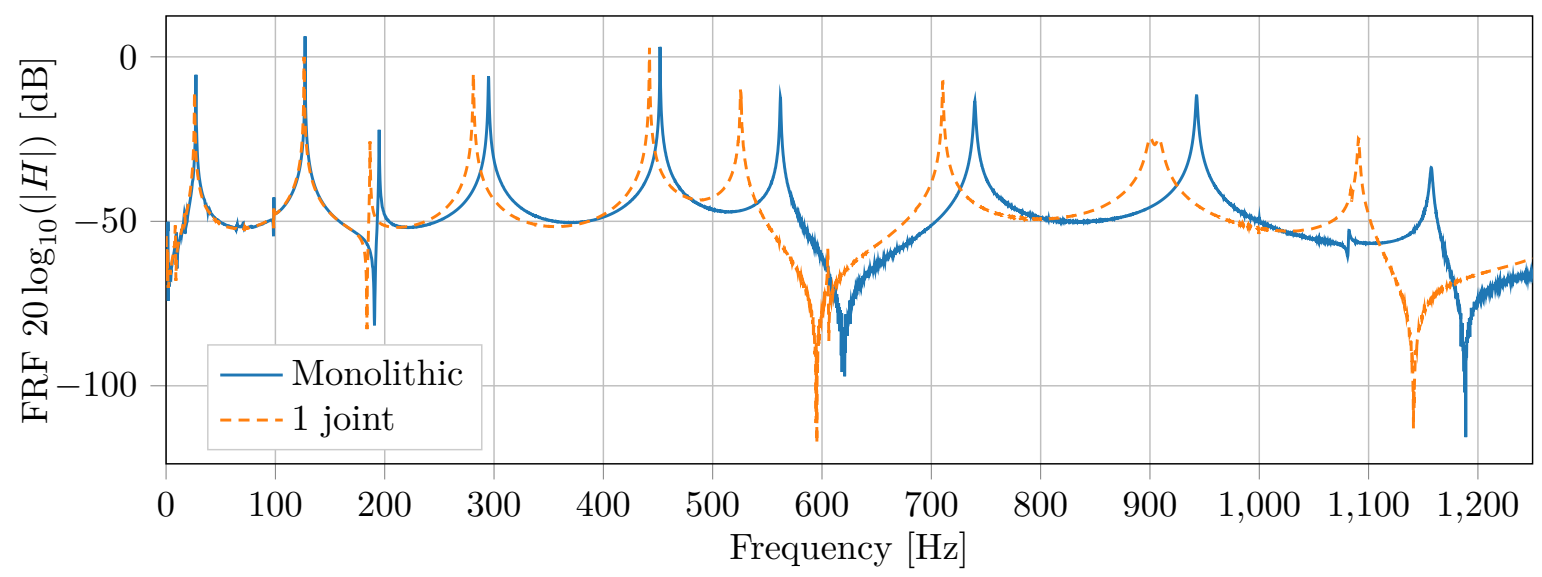

Figure 6: Experimental FRF of the monolithic frame and of the frame with a single bolt.

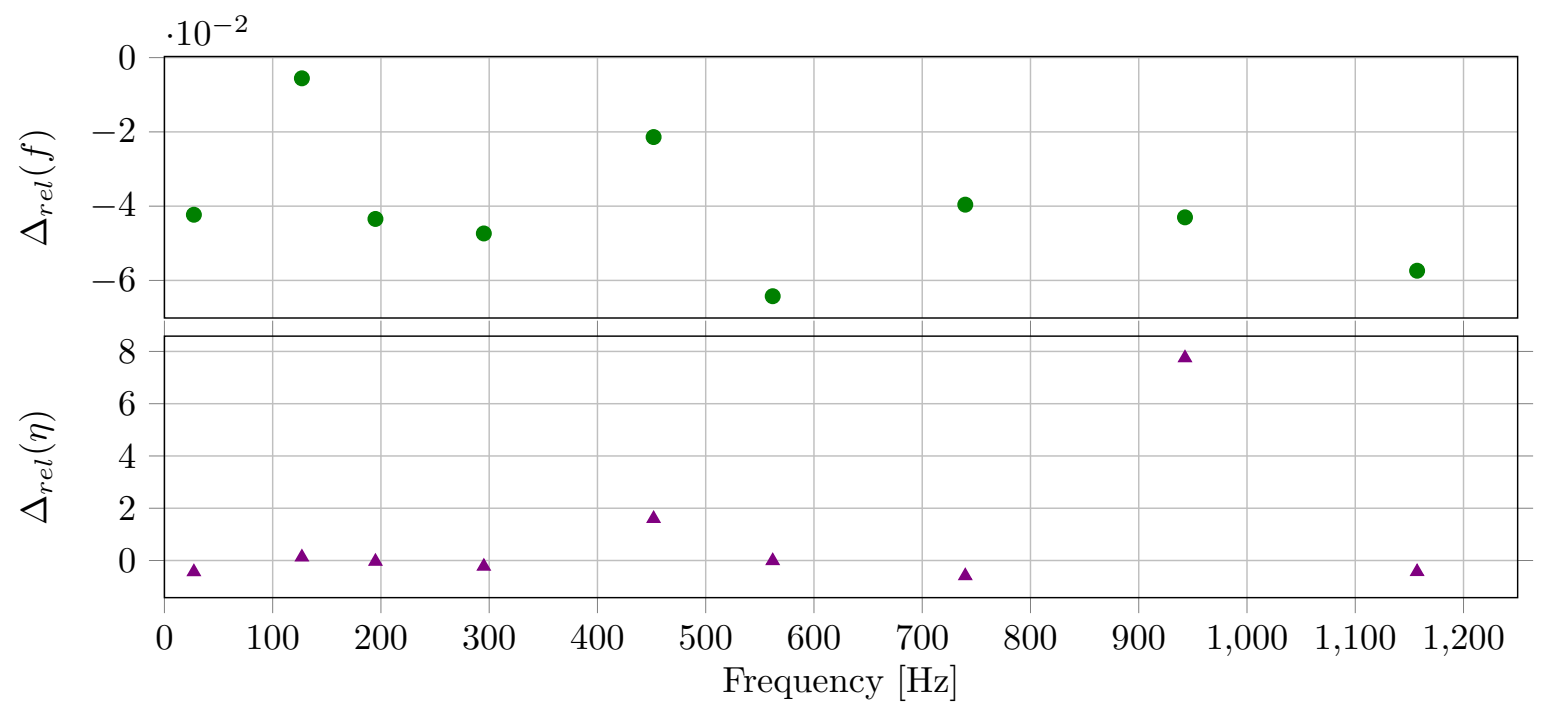

Figure 7: Relative variations of the eigenfrequencies and of the damping ratio associated to the first eigenmodes of the frame with only one bolt compared with the monolithic frame. 
are compared on Figure 6. As expected, the bolted joint affects the dynamical characteristics of the assembly: a decrease of its eigenfrequencies is observed; the joint stiffness increases with the tightening toward a limit value. The excitation magnitude tends to soften the joint. This is due to the slipping area in the joint interface which increases with the excitation magnitude and decreases with the joint tightening [59]. Similar results can be found in the literature, for example [36, 60]. The experimental observations also highlight a particular dependency of the joint stiffness to the frame's eigenfrequencies as illustrated on Figure 7. This figure illustrates the relative variation of eigenfrequencies of the assembled frame with only one bolt and the monolithic one. It also shows the relative variation of the modal damping ratio associated to each mode. The modal damping factors are identified using the classical Nyquist circle-fit-method [61]. It can be noticed that the damping induced by the joint is negligible in most cases except for modes 5 and 8. For this reason, this work is limited to the modelling of the joint stiffness. The modal damping identified experimentally is used to compute the numerical FRF of the frame.

For each eigenfrequency, the associated eigenshape differs from the others, which results in a different loading configuration of the joint and, as a consequence, in a different joint stiffness. For the considered frame, the joints are mostly loaded in bending. The joint stiffness model should consequently depend on the local curvature of the frame eigenshapes. Quantitatively, the observed shift of the bolted frame eigenfrequencies with the magnitude holds below one percent which illustrates a weak non-linearity of the behaviour of the assembled structure. A linear macroscopic model of joint seems therefore sufficient, the variability due to the excitation magnitude would be taken into account through the stochastic extension of this model. The observed behaviour of the bolted frame leads us to also model the variability due to the bolt tightening through the stochastic model.

The studied structure is made of slender parts, a beam finite element model is then adapted to simulate its vibrational behaviour. Numerous interface models can be found in the literature as mentioned on Section 2.1. For beam like structures, node-to-node elements are well adapted. Experimental results indicate that the sought model should directly relate the joint stiffness to the local curvature of the structure. By definition, the bending deformation energy of a beam depends directly on the curvature and is proportional to $Y I$, where $Y$ is the Young's modulus and $I$ the area moment of inertia associated to bending. The element's nodes belonging to each assembled part in the joint area are linked using node-to-node rigid connections. The joint stiffness is modeled through an equivalent Young's modulus applied to the beam elements in this area as illustrated on Figure 3c.

To identify the Young's modulus of the chosen nominal model, we choose to minimize the least square error using a simplex-based minimization algorithm, proposed on Equation (1), between the first $N$ experimental $\left(f_{k}^{\exp }\right)$ and simulated eigenfrequencies $\left(f_{k}^{\mathrm{EF}}\right)$ of the frame.

$$
\varepsilon_{l s}=\sum_{k=1}^{N}\left(f_{k}^{\exp }-f_{k}^{\mathrm{EF}}\right)^{2} .
$$

Figure 8 illustrates the nominal FRF of the frame simulated with the finite element model defined on Figure $3 \mathrm{c}$ and the identified Young's modulus $(Y=11.5 \mathrm{GPa})$ in the joint area. Figure 2 shows the relative error between experimental eigenfrequencies and eigenfrequencies simulated with the identified Young modulus. The nominal model of the bolted joint is then just sufficient to simulate the behaviour of a frame with a single bolt on its vertical beam. It might be reminded that the previous development was applied to a bolted joint linked to a vertical beam of the studied frame. As a bolt positioned on the horizontal beam would not necessarily be loaded in a similar manner, the identification process and the needed experimental results have been reiterated for this second bolt configuration. Similar results are obtained and, for simplicity's sake, would not be presented here. To illustrate the dynamic behaviour of the fully bolted frame, the first ten eigenshapes of the frame simulated with these two deterministic models of joint are illustrated on Figure 9 .

A simple nominal model of a joint has been defined and identified for the studied assembled frame. This model will be extended in order to define and identify a stochastic model of a joint. The next Section aims to define an appropriate framework to characterize the vibrational behaviour of an assembled structure considering the variability of the joint's parameters. 


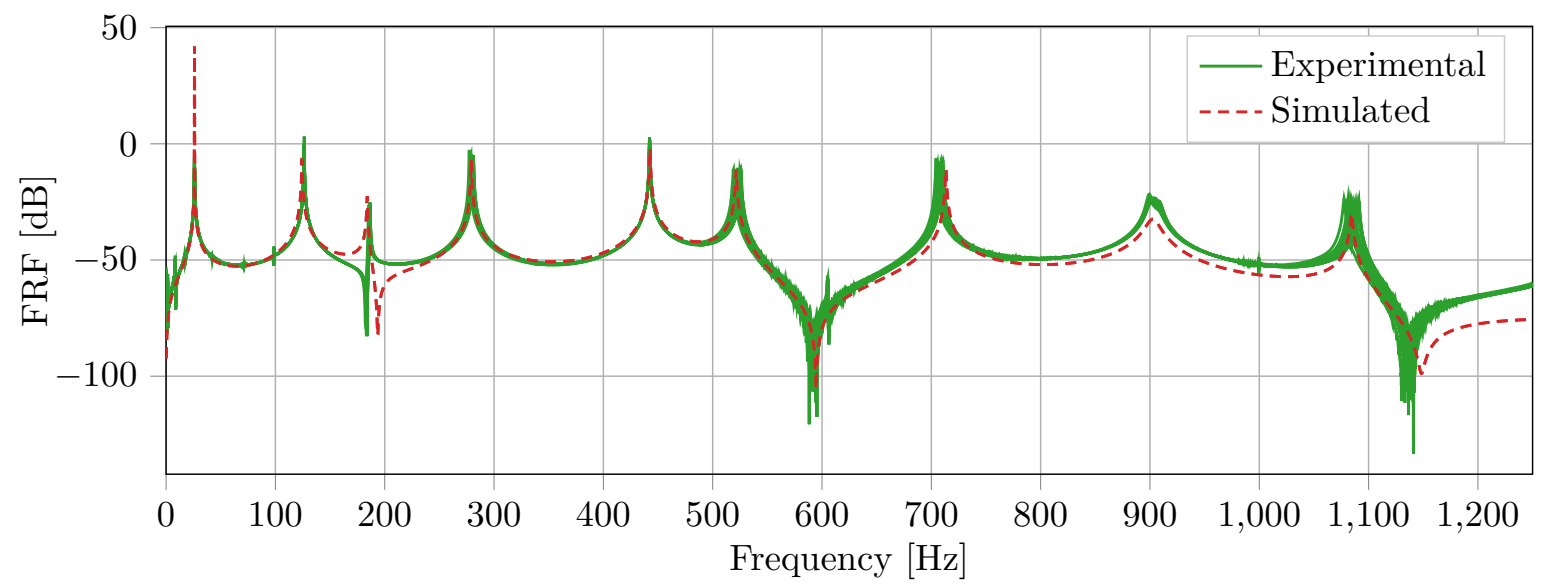

Figure 8: Experimental and simulated FRF of the frame with a single bolt.

\begin{tabular}{|l||c|c|c|c|c|c|c|c|c|}
\hline Index of freq. & 1 & 2 & 3 & 4 & 5 & 6 & 7 & 8 & 9 \\
\hline \hline$f_{\text {exp }}(H z)$ & 26.00 & 126.26 & 186.06 & 279.70 & 442.28 & 523.25 & 708.10 & 902.36 & 1086.27 \\
\hline$f_{\text {simu }}(H z)$ & 26.00 & 124.25 & 184.00 & 279.25 & 442.75 & 521.50 & 713.25 & 902.50 & 1084.25 \\
\hline$\epsilon_{\text {rel }}$ & $<10^{-3}$ & 0.016 & 0.011 & 0.0016 & 0.0011 & 0.0033 & 0.0073 & $<10^{-3}$ & 0.0018 \\
\hline
\end{tabular}

Table 2: Relative error between identified and experimental eigenfrequencies of the frame with only one bolt.
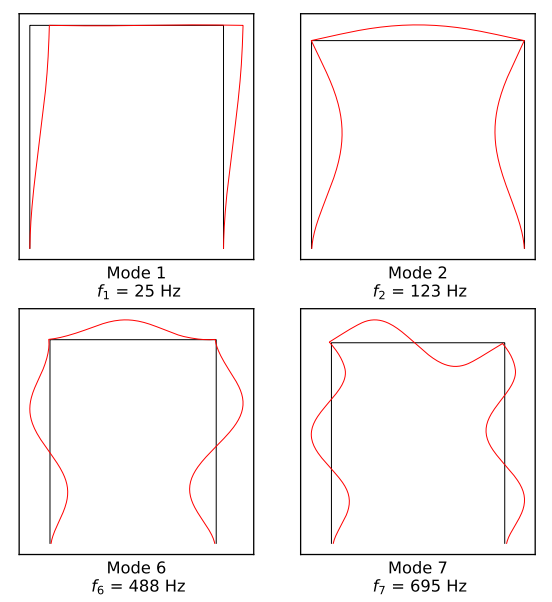

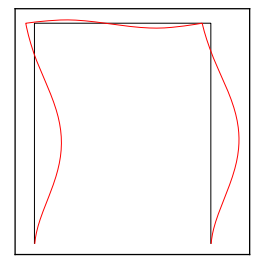

Mode 3
$f_{3}=178 \mathrm{~Hz}$

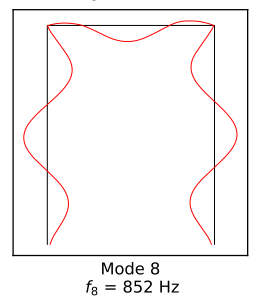

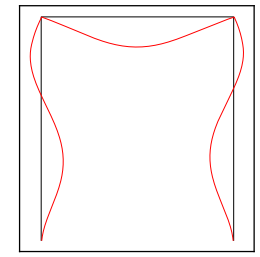

Mode 4
$f_{4}=272 \mathrm{~Hz}$

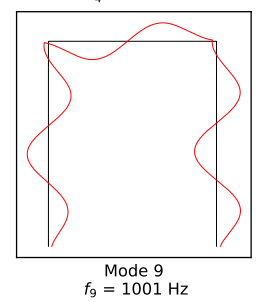

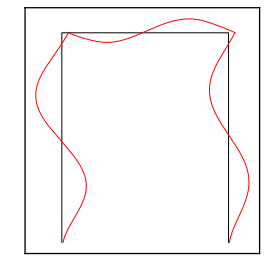

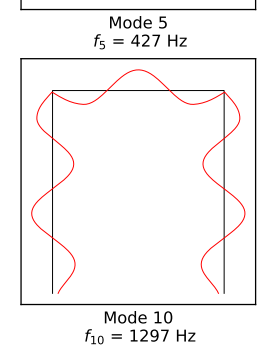

Figure 9: First ten eigenshapes of the studied frame (fully bolted). 


\section{Uncertainties for assemblies on the context of vibration}

\subsection{State of art}

Methods for propagation of uncertainty can be divided into two categories depending on whether they are probabilistic or not. Non-probabilistic approaches as fuzzy logic [62], interval approaches or lackof-knowledge theory [19, 23, 58] are justified for epistemic and random uncertainties. Nevertheless, probabilistic approaches aim to model the natural variability of random phenomena. Two branches can be identified. On the one hand, parametric probabilistic approaches aim to model the variability of the parameters of a given model. On the other hand non-parametric probabilistic approaches intend to take into account uncertainties due to measurement or to the model itself [10]. The parametric probabilistic approach consists in modeling the uncertain parameters of the computational model by random variables and then in constructing the stochastic model of these random variables using the available information. Such an approach is very well adapted and very efficient to take into account the uncertainties on the computational model parameters as soon as the probability theory can be used. Nevertheless, the parametric probabilistic approach does not allow the modeling uncertainties to be taken into account. Two main methods can be used to take into account modeling uncertainties (modeling errors). The first one consists in introducing a stochastic model of the output-prediction-error, considered as a noise, which is the difference between the real system output and the computational model output. Such an approach is simple and efficient but requires a lot of experimental data in high dimension. The second one is based on the non parametric probabilistic approach of modeling uncertainties (modeling errors) based on the use of a reduced-order model and the random matrix theory. It consists in directly constructing the stochastic modeling of the operators of the mean computational model using the Maximum Entropy Principle. Concerning the coupling of the parametric probabilistic approach of the uncertainties on the computational model parameters with the nonparametric probabilistic approach of modeling uncertainties (modeling errors), a methodology has been proposed in computational mechanics $[10,11,63]$. This generalized probabilistic approach of uncertainties in computational dynamics uses the random matrix theory. The proposed approach allows the prior stochastic model of each type of uncertainties (uncertainties on the computational model parameters and modeling uncertainties) to be separately constructed and identified. The modeling errors are not taken into account with the usual output-prediction-error method but with the nonparametric probabilistic approach.

In the context of this paper, the substructures (parts) of the assembly are supposed to be well-known: they are modelled by beams or plates of elastic material, therefore the associated model is supposed sufficiently accurate and its characteristics are supposed deterministic. To take into account the variability of the actual behaviour of the joints, the parametric probabilistic framework is then adopted $[12,64]$. To solve a dynamical problem in a probabilistic framework, methods based on statistical sampling, also referred to as Monte-Carlo methods, are straightforward, easy to implement and above all the most accurate methods as they statistically converge towards the exact solution of the stochastic problem [65, 66]. Even if they are also independent of the stochastic dimension of the problem and they allow to consider any level of variability for the considered parameters [67], the computational cost necessary to ensure the convergence of the result drastically increases with the computational cost of a single deterministic computation of the studied structure. Numerous techniques, generally based on variance reduction or on computation parallelization, have been developed [68], nevertheless for structural analysis these methods remain limited. As an alternative, non sampling methods have been developed in the last decades. Two particular approaches are mainly used in the literature to approximate the statistical properties of the response of a random system: the perturbation and the spectral methods $[66,69,70]$.

The Perturbation Method [69, 71, 72] is based on an approximation of the random variables of interest through the truncation of their Taylor expansion while the Spectral Stochastic Finite Element Method (SSFEM), introduced by Ghanem and Spanos [66] and inspired by Wiener's works [73], is based on a discretization of the random variables of interest among a finite random space. The implementation of the Perturbation Method is pretty easy, nevertheless application of this expansion is generally limited to the second order and variations of the system parameters should remain small to guarantee the accurate 
estimation of statistical moments [69]. Recently, new approaches propose to use together the component mode synthesis and probabilistic uncertainty methods considering Perturbation Methods[74-76]. The SSFEM is based on the representation of any random variable in a basis of orthogonal polynomials of Gaussian Random variables called the Polynomial Chaos (PC). The coefficients of the decomposed random variable could be estimated using a Galerkin-based method and a Monte-Carlo sampling as described in [77]. This simulation-based method becomes quickly computationally intensive. Ghanem and Ghosh also propose to reduce the problem to a set of deterministic non-linear equations using an other Galerkin method. Alternatives to these intrusive approaches have been proposed to compute the response of a stochastic model using only calls to deterministic model. Some usual non-intrusive approaches based on PC expansion are presented by Blatman and Sudret [78]. Nevertheless, methods based on PC expansion appear to be complex to implement and require intensive computations in the case of high-order polynomials or a high number of random parameters. Sparse Polynomial Chaos method has been proposed by Blatman and Sudret [78] to decrease the computational time in a nonintrusive manner, nevertheless PC based methods remain complex to implement. In the context of this work, we choose to use a non-intrusive method referred to as the Stochastic Model Reduction (SMR) approach [27]. This approach benefits from simple implementation as perturbation methods and allows to consider random variables not limited to small variations. This approach will be briefly introduced in the following Section.

\subsection{Stochastic Model (SMR)}

This Section gives a short overview of the Stochastic Model Reduction approach referred hereafter as SMR approach. More details about the basic assumptions of this approach, comparisons with other classical methods and practical applications are available in the dedicated paper [27] or in [28].

The general eigenvalue problem of undamped systems can be expressed for a problem with $n_{d}$ degrees of freedom by:

$$
\lambda_{k}(\theta) \mathbf{M}(\theta) \boldsymbol{\Phi}_{k}(\theta)=\mathbf{K}(\theta) \boldsymbol{\Phi}_{k}(\theta) .
$$

$\lambda_{k} \in \mathbb{R}$ and $\boldsymbol{\Phi}_{k} \in \mathbb{R}^{n_{d}}$ are respectively the $k$ th eigenvalue and the $k$ th associated eigenvector.

The eigenvector $\boldsymbol{\Phi}_{k}$ is assumed to be mass normalized such that $\boldsymbol{\Phi}_{k}^{\top} \mathbf{M} \boldsymbol{\Phi}_{k}=1$. Matrices $\mathbf{M}(\theta) \in$ $\mathbb{R}^{n_{d} \times n_{d}}$ and $\mathbf{K}(\theta) \in \mathbb{R}^{n_{d} \times n_{d}}$ represent the mass and stiffness matrices of the structure. Their randomness is due to the physical parameters of the structure such as mass density, Young's modulus or geometric properties. $(\Omega, \mathcal{F}, p)$ is the abstract probability space associated with the underlying physical experiments. $\theta \in \Omega$ is a basic event from the complete probability space $\Omega$. The space of square integrable random variables is denoted by $L_{2}(\Omega)$ and forms a Hilbert space with the norm $\|\cdot\|_{L_{2}(\Omega)}$. In this paper, $\mathrm{E}[\cdot], \operatorname{Var}[\cdot]$ and $\sigma[\cdot]$ denote respectively the mathematical expectation, variance and standard deviation.

The SMR approach is presented here considering the case of an assembled structure composed of $n$ sub-structures with $n$ different Young's moduli $\left(Y_{1}, Y_{2}, \ldots, Y_{n}\right)$. This case well fits the equivalent stiffness model of a bolted joint presented in the previous Section. Nevertheless, this formulation could also correspond to other problems, for example, a metallic structure provided with piezoelectric patches or a multi-materials glued structure. The Young's moduli are assumed to be the only random parameters in the considered problem. Thus the stiffness matrix $\mathbf{K}(\theta)$ is a random matrix and $\mathbf{M}$ remains deterministic. The global stiffness matrix can be written according to different stiffness matrices relating to each sub-structure:

$$
\mathbf{K}(\theta)=\sum_{i=1}^{n} \mathbf{K}_{i}(\theta)=\sum_{i=1}^{n} \gamma_{i}(\theta) \mathbf{K}_{i},
$$

where $\gamma_{i}(\theta)$ is the random parameter corresponding to the $i$ th Young's modulus and defined as:

$$
\gamma_{i}(\theta)=\frac{Y_{i}(\theta)}{Y_{0 i}},
$$

where $Y_{0 i}=\mathrm{E}\left[Y_{i}(\theta)\right]$ and $\mathrm{E}\left[\gamma_{i}(\theta)\right]=1 . \mathbf{K}_{i}$ are the sensitivity stiffness matrices of the global stochastic stiffness matrix $\mathbf{K}(\theta)$ with respect to the parameter $\gamma_{i}(\theta)$. With this notation the stochastic behavior 
is only taken into account by the coefficient $\gamma_{i}(\theta)$. These $\mathbb{R}^{n_{d} \times n_{d}}$ matrices correspond in this linear case to a global sub-structure stiffness matrix obtained with the mean value of the Young's modulus (stiffness matrix of the sub-structure reshaped in the whole basis of degrees of freedom of the considered problem). In addition, $\mathbf{K}_{i}(\theta)$ are the global sub-structure stochastic stiffness matrices.

The first assumption of the SMR approach is: considering a structure with random parameters, its eigenvalues are random but the corresponding eigenvectors are nearly deterministic. Then it is assumed that Young's moduli variations around an expected value do not change the structure eigenshapes. Therefore, the matrix of random eigenvectors corresponds to the matrix of eigenvectors calculated with the expected value parameters $\boldsymbol{\Phi}(\theta)=\overline{\boldsymbol{\Phi}}$. Thus, this assumption added to the mass normalization of eigenvectors $\boldsymbol{\Phi}_{k}$ allows us to rewrite the $k$ th random frequency as:

$$
\lambda_{k}(\theta)=\left(2 \pi f_{k}(\theta)\right)^{2}=\sum_{i=1}^{n} \gamma_{i}(\theta) \overline{\mathbf{\Phi}}_{k}^{\top} \mathbf{K}_{i} \overline{\mathbf{\Phi}_{k}}=\sum_{i=1}^{n} \lambda_{k i} \gamma_{i}(\theta) .
$$

The coefficients $\lambda_{k i}$ of this expansion are deterministic. The randomness of the eigenvalue is only due to the input random parameters. As a consequence, this first refinement level enables us to obtain a closed form of the approximate eigenvalue for which the two first central moments of the random eigenvalues are obtained with no computational cost.

When the variability of the eigenshapes increases, it is no longer possible to approximate the random eigenvectors as deterministic eigenvectors. In order to accurately estimate the corresponding eigenvalues it is proposed to take into account the eigenshapes variability with a Taylor expansion about the point $\gamma(\theta)=\gamma_{0}$. This improvement is referred hereafter as SMR2.

The $k$ th random eigenvalue $\lambda_{k}(\gamma(\theta))$ estimated using SMR2 can be written as:

$$
\begin{aligned}
\lambda_{k}(\theta) & =\sum_{i=1}^{n} \gamma_{i}(\theta) \boldsymbol{\Phi}_{k}^{\top}(\gamma(\theta)) \mathbf{K}_{i} \boldsymbol{\Phi}_{k}(\gamma(\theta)), \\
\boldsymbol{\Phi}_{k}(\gamma(\theta)) & =\boldsymbol{\Phi}_{k}\left(\gamma_{0}\right)+\left.\sum_{i=1}^{n} \frac{\partial \boldsymbol{\Phi}_{k}}{\partial \gamma_{i}}\right|_{\gamma_{i}=1}\left(\gamma_{i}(\theta)-1\right) .
\end{aligned}
$$

For details on the computation of the derivatives of each eigenvector, reader could refer to [27, 28].

For actual structures, the set of random eigenvalues will contain either eigenvalues corresponding to almost deterministic eigenvectors and eigenvalues corresponding to random eigenvectors. The two refinement levels of the SMR approach have to be jointly used to ensure the best estimation of the whole set of random eigenvalues. To this purpose, a simple resolution approach is summarized on Figure 10. This approach consists in adapting the invested computational resources to each random eigenvalue. First, all random eigenvalues are approximated with SMR1, assuming that all eigenvectors are deterministic. This first step provides an initial estimation of all eigenvalues. The next step consists in the validation of the SMR1 assumption. If not, it is necessary to refine the approximation. In the case of well separated eigenvalues, the eigenshape sensitivity with respect to the input parameters is small, the SMR1 assumption is then validated and the corresponding random eigenvalues is accurately estimated with SMR1. In the case of random eigenvalues neither clearly identified nor well separated, SMR2 is used to estimate a better approximation of the eigenvalues. Thus, the SMR2 method is applied on a reduced set of eigenvalues. To decide when the method should be refined, an indicator referred to as Proximity Factor is proposed. Computationally free, this indicator is based on the first two statistical moments of the random eigenfrequencies. Details on this indicator could be found in the dedicated paper [27].

SMR approach has been widely tested in [27] on different test cases. In particular, the approach has been applied on an industrial structure modeled with a commercial software: the Ariane 5 payload adapter. This example illustrates the SMR efficiency in comparison with Monte-Carlo simulation on a 20000 dof finite element model. The SMR approach has been implemented using Python language and coupled with MSC Nastran. The first two statistical moments of the payload adapter first ten random eigenfrequencies are accurately estimated with a computational time reduction from $16 \mathrm{~h}$ to $18 \mathrm{~min}$ (i.e. a time reduction of $98 \%$ ). 


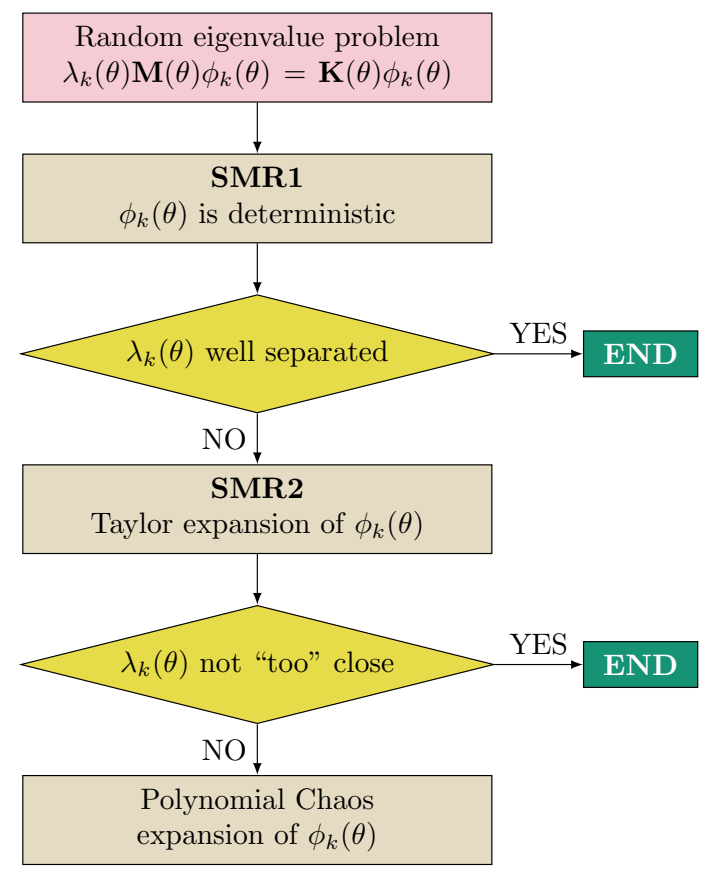

Figure 10: SMR approach for eigenvalue problem resolution.

The SMR approach will be used in the next Section in order to propose an efficient strategy to identify a stochastic model of joint.

\section{Identification of a stochastic model of joint}

This Section aims to define and identify a stochastic model of joint. This model can be defined by randomizing the parameter of the chosen nominal model of joint. This model is therefore defined as a random variable with an unknown distribution. A straightforward approach is used hereafter to characterize the probability density function (pdf) associated to this random variable.

\subsection{Identification process with uncertainties}

Non-parametric methods, for instance kernel-based methods [16, 79], could be used to estimate the pdf of a random variable as soon as a relatively large set of samples is available. In this work, as only a limited amount of data is obtained from experiments, it is necessary to define a parametrized distribution associated to the unknown random variable. An expansion-type representation of the random variable could be employed, for instance using a polynomial chaos expansion, or the maximum entropy principle could be used as a more straightforward approach $[10,16]$. This latter approach has been chosen for this work. The identification process of the stochastic model of the joint proposed here is to first identify an appropriate pdf associated to the random variable according to the maximum entropy principle. And, as a second step, the experimental identification of the parameters of the prior probability distribution of the random variable modeling uncertainties of the computational model parameters can be performed, either in minimizing a distance between the experimental observations and the random model observations (least-square method for instance), or in using the maximum likelihood method. In our case we used the first approach for the sake of simplicity.

According to the maximum entropy principle, a real-valued random variable $X$ such as $\operatorname{Supp}(X)=$ ] $0,+\infty\left[, \mathrm{E}[X]=m_{x}\right.$ and $\operatorname{Var}[X]=\sigma_{x}^{2}$ follows a Gamma distribution [80]. The pdf of the random joint stiffness $K_{\text {joint }}$ could therefore be modelled as a random variable with Gamma distribution. The 
parameters of the Gamma distribution $\alpha$ and $\beta$ as $K_{\text {joint }} \rightsquigarrow \operatorname{Gamma}(\alpha, \beta)=\Gamma(\alpha, \beta)$ are then given by

$$
\alpha=\left(\frac{\mathrm{E}\left[K_{\text {joint }}\right]}{\sigma_{K_{\text {joint }}}}\right)^{2} \quad \text { and } \quad \beta=\frac{\sigma_{K_{\text {joint }}}^{2}}{\mathrm{E}\left[K_{\text {joint }}\right]} .
$$

The scaling property of the Gamma distribution, whereby if $Y \rightsquigarrow \operatorname{Gamma}(\alpha, \beta)=\Gamma(\alpha, \beta)$, then for any $c>0, c Y \rightsquigarrow \operatorname{Gamma}(\alpha, c \beta)$, allows us to write the input random variables of the problem $\gamma$ (see Equation (4)) as a function of the coefficient of variation $\delta=\sigma_{K_{\text {joint }}} / \mathrm{E}\left[K_{\text {joint }}\right]$ of the joint stiffness:

$$
\gamma \rightsquigarrow \operatorname{Gamma}\left(\frac{1}{\delta^{2}}, \delta^{2}\right) .
$$

The expectation and the standard deviation of the random variable to be identified are then sufficient to completely define its pdf. The identification process of the proposed stochastic model seeks to identify the first two statistical moments of the random joint stiffness corresponding to the observed variability of the dynamical characteristics of the studied structure. In other terms, we seek the first two statistical moments of the input random variable which minimize the error between the statistical moments of the experimental eigenfrequencies of the structure and the eigenfrequencies computed numerically [16].

The computation of the statistical moment samples of the random eigenfrequencies of a structure would require a significant amount of computer resources if they were computed using a Monte-Carlo simulation. This method is thus not affordable for achieving the identification process. The SMR approach, presented on Section 3.2, allows to estimate the first two statistical moments of the random eigenfrequencies of a structure with a good precision and an extremely low computation time. It is therefore adapted to the proposed identification process and will be used in the following.

We define the following error for each statistical moment:

$$
\begin{aligned}
\varepsilon_{E} & =\sum_{i=1}^{N}\left[\mathrm{E}\left[f_{i}^{\exp }\right]-\mathrm{E}\left[f_{i}^{\mathrm{SMR}}\right]\right]^{2}, \\
\varepsilon_{\delta} & =\sum_{i=1}^{N}\left[\delta\left[f_{i}^{\exp }\right]-\delta\left[f_{i}^{\mathrm{SMR}}\right]\right]^{2},
\end{aligned}
$$

where $\varepsilon_{E}$ is the error on the expectation of the frame eigenfrequencies, $\varepsilon_{\delta}$ the error on the coefficient of variation and $N$ the number of considered eigenfrequencies of the frame. Notice that the experimental setup has been designed to favour inplane vibrations and the numerical model has been defined and updated to accurately represent the actual structure. Especially boundary conditions, excitation and localized masses have been precisely modelled [28]. Thus, there is no ambiguity in the correspondence between experimental and computed modes which has been checked using a laser scanning vibrometer. The stochastic model identification could then be performed in two steps. First, the expectation of the random variable is obtained minimizing the error $\varepsilon_{E}$. Then the coefficient of variation is computed using this expectation and minimizing $\varepsilon_{\delta}$. Finally, according to Equation (9), the scaling property of the Gamma distribution allows to define the parameters of the Gamma distribution as a function of the statistical moments of the random variable. This completes the characterization of the input random variable.

\subsection{Identification of the stochastic model of joint}

The identification process of the stochastic model of joint seeks to identify the first two statistical moments of the random joint stiffness corresponding to the observed variability of the dynamical characteristics of the single bolted frame. The variability of the eigenfrequencies of a single bolted frame has been obtained experimentally using the experimental setup presented Section 2.2. To that end, the tightening load of the bolt and the structure excitation magnitude have been considered as being the two predominant parameters influencing the joint dynamical behaviour. This identification procedure has been applied for a configuration of the frame with only one bolt linked to its vertical beam as 
Martin Ghienne, Luc Laurent \& Claude Blanzé. Robust characterization of the vibrational behaviour of light assembled structures with random parameters. Mechanical Systems and Signal Processing, 136, 2020. doi: 10.1016/j.ymssp.2019.106510

\begin{tabular}{|l||c|c|c|c|c|c|c|c|c|}
\hline Index of freq. & 1 & 2 & 3 & 4 & 5 & 6 & 7 & 8 & 9 \\
\hline \hline $\mathrm{E}\left[f_{\exp }\right]$ & 26.00 & 126.26 & 186.06 & 279.70 & 442.28 & 523.25 & 708.10 & 902.36 & 1086.27 \\
\hline$\sigma\left[f_{\exp }\right]$ & 0.095 & 0.016 & 0.46 & 1.29 & 0.075 & 2.44 & 2.14 & 0.86 & 4.66 \\
\hline$\delta\left[f_{\exp }\right]$ & 0.0036 & 0.00013 & 0.0025 & 0.0046 & 0.00017 & 0.0047 & 0.0030 & 0.00096 & 0.0043 \\
\hline
\end{tabular}

Table 3: Variability of the experimental eigenfrequencies of the frame with a single bolt.

presented in Section 2.2. The experimental variability has been obtained by varying the bolted joint preload between the lowest and the strongest tightening levels defined in Section 2.2.1. Nine tightening levels have been considered. They are chosen to be non-linearly distributed in order to maximise the variability of the equivalent joint stiffness. More low tightening levels have been considered as the equivalent stiffness of the joint varies more when the joint interface is not fully sticked. This tightening level distribution allows us to span the entire range of the possible joint stiffness values with a relatively small number of experiments. The statistical moments of the experimental eigenfrequencies (required for the identification process presented on Section 4.1) are then computed from these experimental samples and are presented in the Table 3.

In order to be able to characterize configurations of frame with more than one bolt, the case of a single bolted frame with a bolt on its horizontal beam has been also considered. Results are shown on Table 4 where index $J_{\text {vert }}$ refers to joints associated to a vertical beam of the frame and index $J_{\text {hor }}$ to joint on the horizontal beam. It can be noticed that the Young's modulus associated to the vertical joint is significantly lower than the Young's modulus of the material of the rest of the structure while the Young's modulus associated to the horizontal joint is of the same order of magnitude as the frame material. This can be explained by the different loading configurations of each joint.

\begin{tabular}{|l|c|c||c|c|}
\hline \multirow{2}{*}{$\begin{array}{l}\text { Statistical moment } \\
\text { of the joint stiffness }\end{array}$} & $\mathrm{E}\left[Y_{J_{\text {vert }}}\right](\mathrm{Pa})$ & $\delta\left[Y_{J_{\text {vert }}}\right]$ & $\mathrm{E}\left[Y_{J_{\text {hor }}}\right](\mathrm{Pa})$ & $\delta\left[Y_{J_{\text {hor }}}\right]$ \\
\cline { 2 - 5 } & $24 \times 10^{9}$ & 0.09 & $73.5 \times 10^{9}$ & 0.22 \\
\hline \hline \multirow{2}{*}{$\begin{array}{l}\text { Gamma distribu- } \\
\text { tion parameters }\end{array}$} & $\alpha_{\gamma_{1}}$ & $\beta_{\gamma_{1}}$ & $\alpha_{\gamma_{2}}$ & $\beta_{\gamma_{2}}$ \\
\cline { 2 - 5 } & 123 & 0.0081 & 20.7 & 0.0484 \\
\hline
\end{tabular}

Table 4: Parameters of the joints stochastic models ( $J_{\text {vert }}$ and $J_{\text {hor }}$ correspond to the joints respectively on the vertical and the horizontal beams of the frame). Reminder : the Young modulus of the other parts of the structure is $Y_{\text {alu }}=65.5 \mathrm{GPa}$

\subsection{Validation of the identification procedure}

An a posteriori validation of the quality of the SMR results has been performed in order to ensure that, for the identified parameters mechanical random variables, the vibrational behaviour of the frame belongs to the validity domain of the SMR approach. The relative error of the first two statistical moments for the first ten random eigenfrequencies of the frame with only one bolt has been computed. The reference statistical moments have been computed from a 10000 sample Monte Carlo simulation. Figure 11 shows this relative error for the two refinement levels of the SMR approach and for a classical second order perturbation method. The second order perturbation method (referred to as Pert2 on the figure) has been added to illustrate the benefits of the SMR approach on this method. It can be noticed that the expectation is estimated with an error less than a percent for both refinement levels of the SMR approach. The standard deviation is estimated with a maximal error of $2.1 \%$ with SMR1 and less than a percent with SMR2. This can be explained by the relatively low variability of the random stiffness. Its coefficient of variation is indeed less than 0.1. The same validation has been performed for the frame with a single bolt on its horizontal beam. The maximum relative error on the estimation of the expectation of the first ten eigenfrequencies of this second configuration of frame is less than $0.2 \%$ with SMR1 and far less than a percent with SMR2. For the standard deviation, the maximum error is $10 \%$ with SMR1 and $1.8 \%$ with SMR2. It can be concluded that the SMR approach can be used to 
characterize the vibrational behaviour of any configuration of single bolted frame.
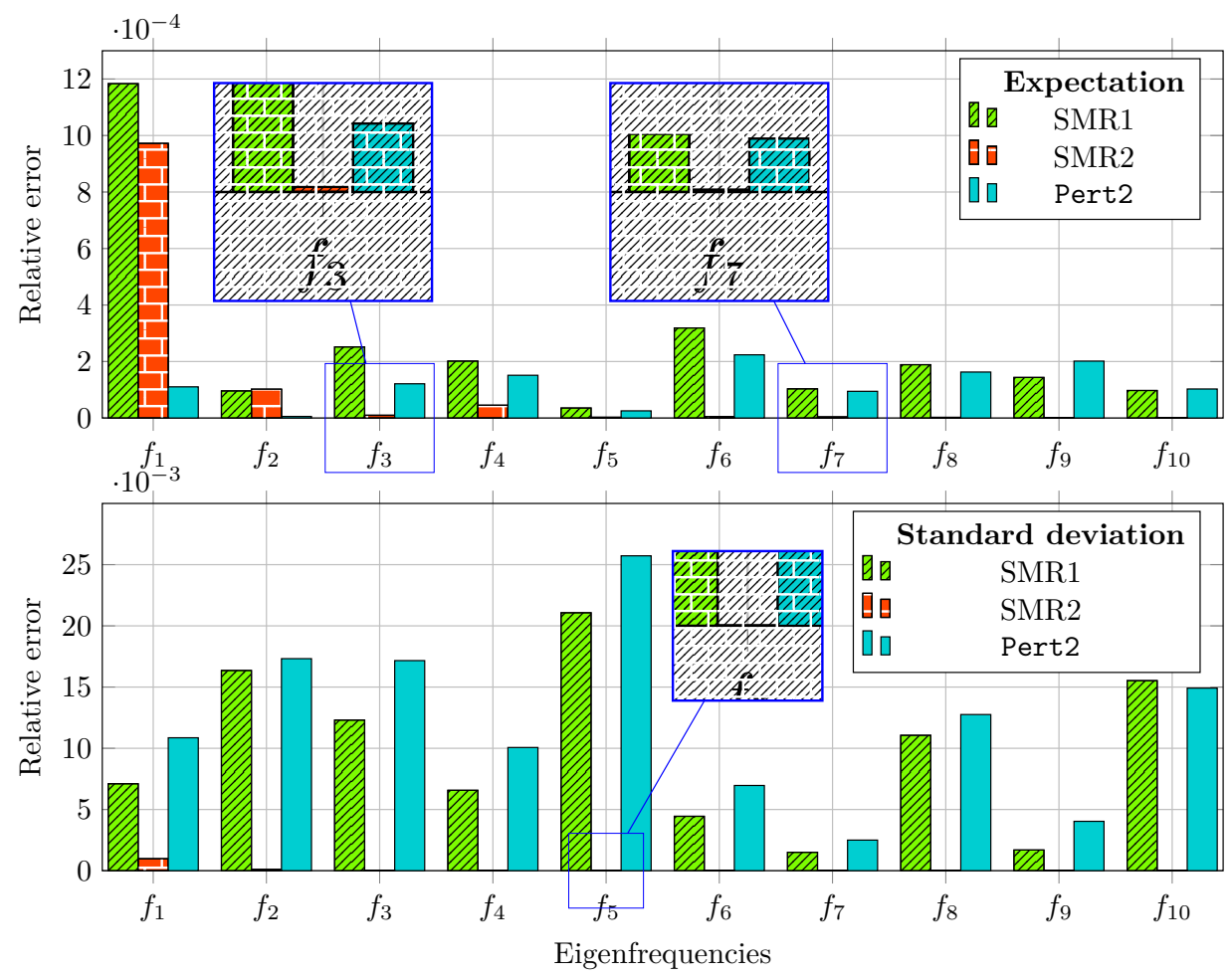

Figure 11: Relative errors on the estimation of the statistical moments of the first ten random eigenfrequencies of the frame with only one bolt on its vertical beam. Pert2 denotes the second order perturbation method.

SMR1 is then used to simulate the vibrational behaviour of a frame with only one bolt taking into account the variability of the joint stiffness. In the remainder of this paper, we choose to present the stochastic FRF of the frames simulated with the SMR approach as follows:

- the mean FRF is plotted with full line, it is computed using the expectation of the random eigenvalues and the deterministic eigenvectors of the frame;

- the envelope curve which embraces the mean FRF is obtained using 10000 sample of the independent random stiffnesses.

Notice that the lack of adapted representation tool has motivated the choice of an envelope curve to illustrate the variability of the vibrational behaviour of the frame. It is however possible to compute the probability of occurrence of each FRF in the envelope curve using the SMR approach if needed.

Using this representation, the experimental and simulated variability of the frame with only one bolt on its vertical beam are presented on Figure 12. It can be noticed that the simulated stochastic behaviour of the frame fits well the variability observed experimentally. A shift of the mean of some eigenfrequencies is however observed for the highest frequencies. This drawback can be attributed either to the chosen beam model of the frame or to the numerical representation of boundary conditions as this misfit is also observed for a monolithic frame. Equivalent results have been obtained for the case of a frame with only one bolt on its horizontal beam. Therefore, this second case would not be developed here. 


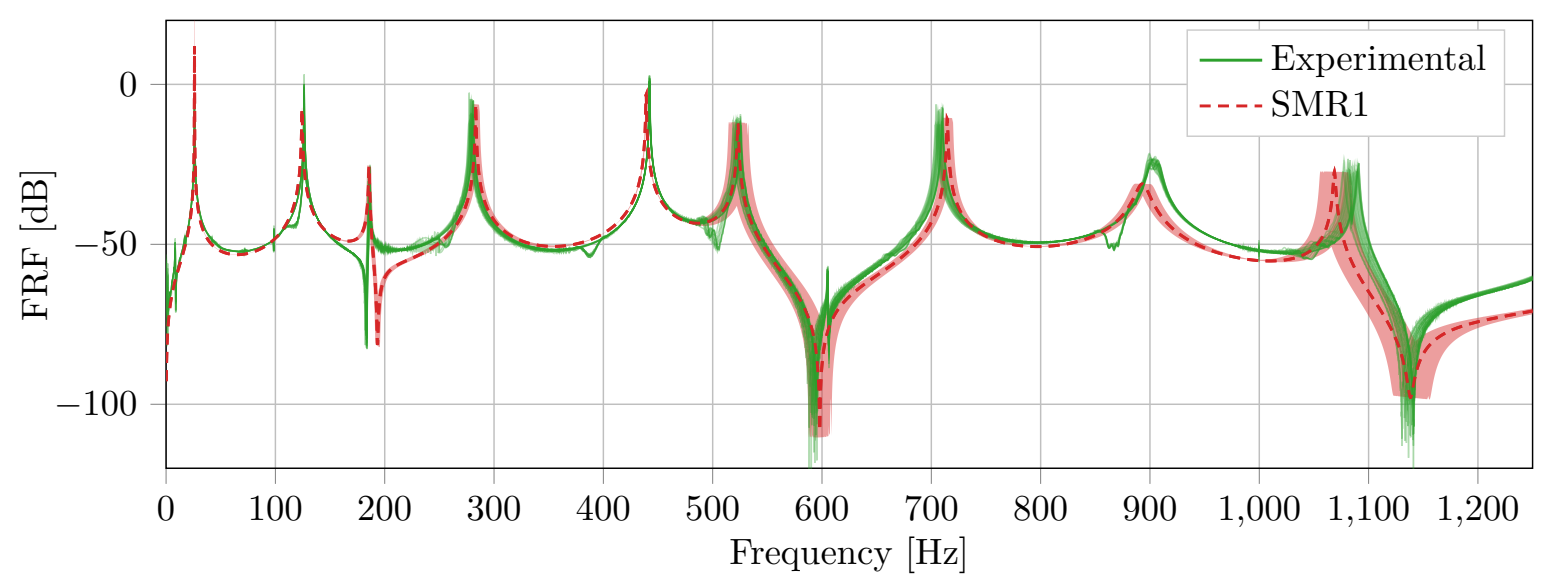

Figure 12: Comparison of the experimental and simulated FRF of a single bolted frame.

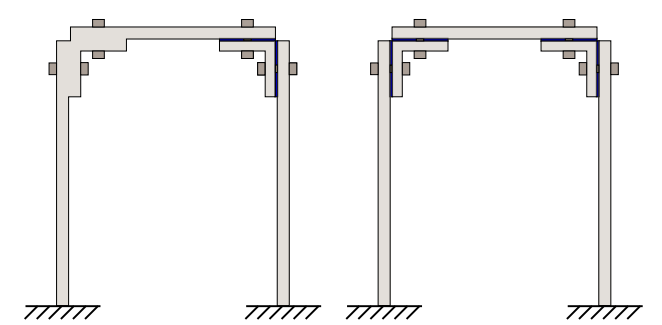

(a)

(b)

Figure 13: Multi-bolted frame configurations.

\section{Robust characterization of the vibrational behaviour of multi-bolted frame}

A stochastic model of joint has been defined and validated for each configuration of joint of the studied frame. Even if the nominal model of joint has raised some limitations, this allows us to robustly characterize the vibrational behaviour of any configuration of frame with several joints. This Section aims to validate the applicability of the stochastic model of joint to multi-bolted frame. In particular, frame with respectively two and four bolts as shown on Figure 13 are concerned here. The stochastic response simulated with the SMR approach and the stochastic models of joint is compared to the experimental variability of the two studied frame in order to validate the proposed approach.

\subsection{Two-bolted frame}

First, the experimental variability of the vibrational behaviour of a two-bolted frame, as defined Figure $13 \mathrm{a}$, is characterized. The tightening of each bolt and the magnitude of excitation of the frame are considered as control parameters. Two magnitudes of excitation are considered and three tightening torque for each bolt are considered corresponding to low, medium and high tightening levels as defined Section 2.2.1. The experimental variability of the frame is obtained using a full factorial experiment considering the three tightening levels (referred from 1 to 3 in Table 5) for each of the two joints (referred to as bolt $\mathrm{A}$ and $\mathrm{B}$ in Table 5) and the two magnitudes of excitation. The influence of the tightening sequence has not been considered in this study to limit the duration of the experimental campaign and because this aspect has already been addressed in the literature [29, 81].

Figure 14 shows the variability of the frame FRF simulated with SMR1 and SMR2. The Young's moduli associated with each joint of the frame are defined as random variables with Gamma distribu- 
Martin Ghienne, Luc Laurent \& Claude Blanzé. Robust characterization of the vibrational behaviour of light assembled structures with random parameters. Mechanical Systems and Signal Processing, 136, 2020. doi: 10.1016/j.ymssp.2019.106510

\begin{tabular}{|l|l||l|l|l|l|l|l|l|l|l|}
\hline & Test index & 1 & 2 & 3 & 4 & 5 & 6 & 7 & 8 & 9 \\
\hline \hline \multirow{2}{*}{ Low magnitude } & Bolt A & 1 & 1 & 1 & 2 & 2 & 2 & 3 & 3 & 3 \\
\cline { 2 - 9 } & Bolt B & 1 & 2 & 3 & 1 & 2 & 3 & 1 & 2 & 3 \\
\hline \multirow{2}{*}{ High magnitude } & Bolt A & 1 & 1 & 1 & 2 & 2 & 2 & 3 & 3 & 3 \\
\cline { 2 - 9 } & Bolt B & 1 & 2 & 3 & 1 & 2 & 3 & 1 & 2 & 3 \\
\hline
\end{tabular}

Table 5: Factorial experiment of the two-bolted frame (Load levels: (1) low, (2) medium and (3) high).

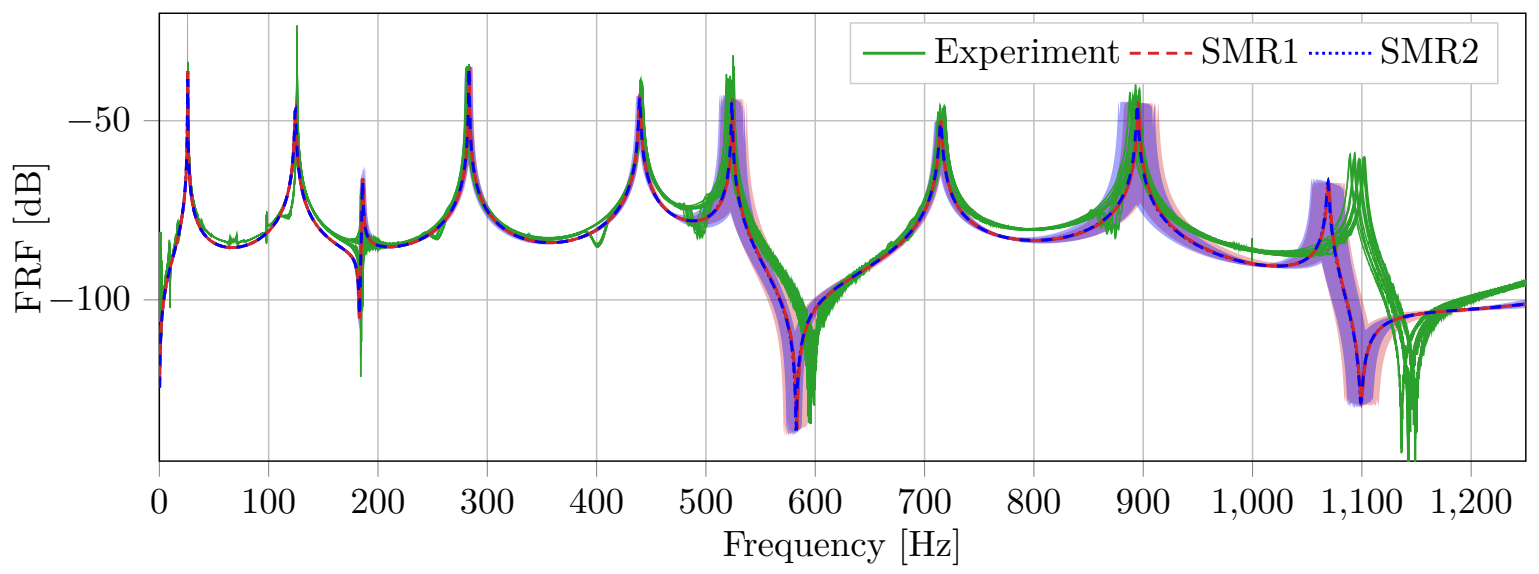

Figure 14: Comparison of the experimental variability of the two-bolted frame and the experimental variability computed with the SMR approach.

tions. The first statistical moments of these random variables are settled as $\mathrm{E}\left[Y_{A}(\theta)\right]=24 \times 10^{9} \mathrm{~Pa}$ et $\delta\left[Y_{A}(\theta)\right]=0.09$ for the joint between the frame corner and the vertical beam and $\mathrm{E}\left[Y_{B}(\theta)\right]=$ $73.5 \times 10^{9} \mathrm{~Pa}$ et $\delta\left[Y_{B}(\theta)\right]=0.22$ for the joint between the corner and the horizontal beam. The average FRF (solid line) are computed using the deterministic eigenvectors of the frame and the expectation of the random eigenfrequencies estimated with SMR1 and SMR2. The envelope of these FRF are obtained with 1000 independent samples of the random stiffnesses associated with each joint. It can be noticed that the difference between the results obtained with SMR1 and SMR2 is not significant. This suggests that, as for the single-bolt test case, SMR1 is sufficient for this frame with two bolts. A shift between experimental and simulated nominal eigenfrequencies at high frequency is observed and can be explained again by the limitations of the finite element model of the frame, the numerical representation of the boundary conditions and the simplicity of the mechanical model used for the joint. Despite the use of a very simple model of the assembled structure, the comparison between the simulated results and the experimental variability illustrates that the stochastic simulation of the frame allows to forecast the experimental variability of this frame configuration.

\subsection{Fully bolted frame}

Finally, the case of a fully bolted frame, as presented on Figure 13b, is considered. The experimental variability of the vibrational behaviour of this frame is characterized considering the preload of each bolt and the magnitude of excitation as control parameters. Two magnitude levels defined as Section 5.1 are considered. Due to the number of joints of the fully bolted frame (4 bolts), the number of tightening level considered here is limited to the two levels corresponding to low and high level as defined Section 2.2.1. The high tightening level could be seen as an actual standard assembly configuration and the low level as a defective joint.

Two designs of experiments have been defined depending on the magnitude of excitation of the frame. The low excitation magnitude is defined as the maximum magnitude ensuring a linear behaviour of the structure. The experimental measurement of the frame FRF is relatively fast for each configuration 
Martin Ghienne, Luc Laurent \& Claude Blanzé. Robust characterization of the vibrational behaviour of light assembled structures with random parameters. Mechanical Systems and Signal Processing, 136, 2020. doi: 10.1016/j.ymssp.2019.106510

\begin{tabular}{|l||c|c|c|c|c|c|c|c|c|c|c|c|c|c|c|c|c|}
\hline Test index & 1 & 2 & 3 & 4 & 5 & 6 & 7 & 8 & 9 & 10 & 11 & 12 & 13 & 14 & 15 & 16 & 17 \\
\hline \hline Bolt A & 1 & 3 & 1 & 1 & 1 & 3 & 3 & 3 & 1 & 1 & 1 & 3 & 3 & 3 & 1 & 3 & 2 \\
\hline Bolt B & 1 & 1 & 3 & 1 & 1 & 3 & 1 & 1 & 3 & 3 & 1 & 3 & 3 & 1 & 3 & 3 & 2 \\
\hline Bolt C & 1 & 1 & 1 & 3 & 1 & 1 & 3 & 1 & 3 & 1 & 3 & 3 & 1 & 3 & 3 & 3 & 2 \\
\hline Bolt D & 1 & 1 & 1 & 1 & 3 & 1 & 1 & 3 & 1 & 3 & 3 & 1 & 3 & 3 & 3 & 3 & 2 \\
\hline
\end{tabular}

Table 6: Partial factorial experiment used for low magnitude of excitation.

\begin{tabular}{|l||l|l|l|l|l|l|l|}
\hline Test index & 1 & 2 & 3 & 4 & 5 & 6 & 7 \\
\hline \hline Bolt A & 3 & 3 & 3 & 1 & 3 & 1 & 2 \\
\hline Bolt B & 3 & 3 & 1 & 3 & 3 & 1 & 2 \\
\hline Bolt C & 3 & 1 & 3 & 3 & 3 & 1 & 2 \\
\hline Bolt D & 1 & 3 & 3 & 3 & 3 & 1 & 2 \\
\hline
\end{tabular}

Table 7: Partial factorial experiment used for high magnitude of excitation.

of joint tightening. A full factorial experiment defined on Table 6 is then considered. Index 1 and 3 correspond respectively to low and high tightening levels. A configuration with medium tightening (index 2) of each bolt is also considered. On the other hand, the high excitation magnitude is five times higher than the lower one and non-linear phenomena occur. For this reason, the FRF acquisition for high magnitude is much longer and a reduced experimental plan is considered. Defined on Table 7, this experimental plan considers the cases of each bolt tightened with the highest level (index 3) except one. The remaining joint is then assimilated as a defective joint. It is tightened with the lowest level (index 1). The three cases where each bolt has the same tightening level, respectively low, medium and high, are also considered. Again, the tightening sequence has been imposed and is not considered as a source of variability in this application. This does not exclude to consider this parameter for actual industrial applications.

The experimental FRF are presented on Figure 16. The stochastic simulation of the fully bolted frame is performed using the finite element model shown on Figure 15. The equivalent stiffness of the boundary conditions has been identified in a preliminary study. The same applies for the stiffness $k_{S}$ of the stinger used between the shaker and the point of application on the frame. The joint stiffnesses of this frame referred to as $Y_{A}(\theta), Y_{B}(\theta), Y_{C}(\theta)$ and $Y_{D}(\theta)$ are modelled as random variables with Gamma distributions. The first statistical moments of these random variables are settled such as:

- $\mathrm{E}\left[Y_{L 1}\right]=\mathrm{E}\left[Y_{L 4}\right]=24 \times 10^{9} \mathrm{~Pa}$;

- $\mathrm{E}\left[Y_{L 2}\right]=\mathrm{E}\left[Y_{L 3}\right]=73.5 \times 10^{9} \mathrm{~Pa}$;
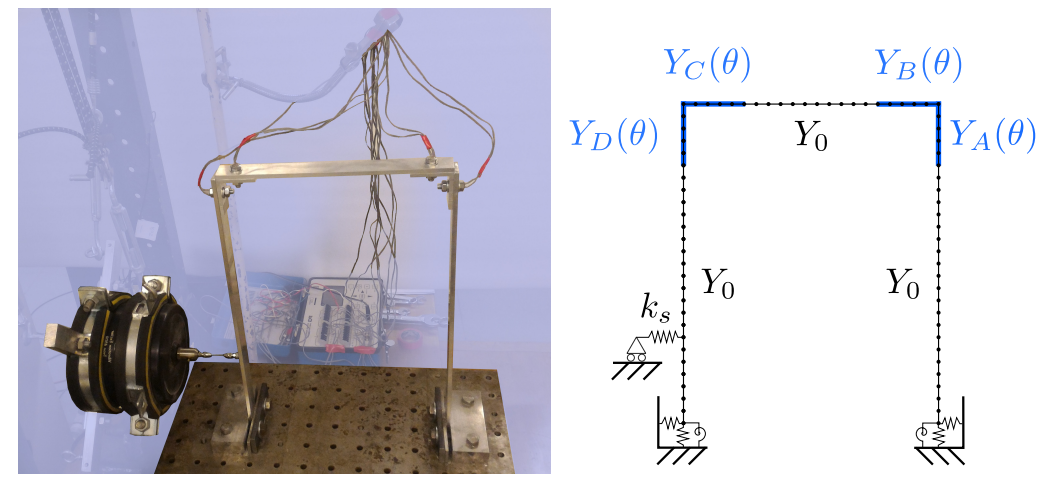

Figure 15: Experimental setup and finite element model of the fully bolted frame. 


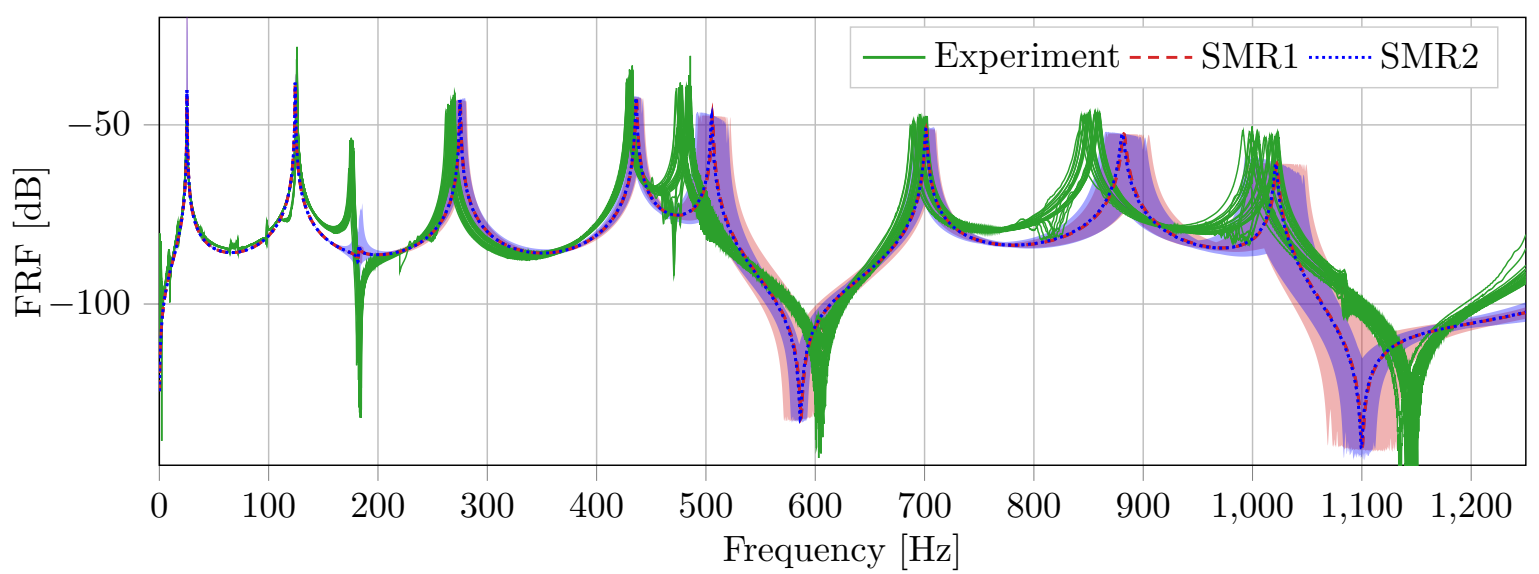

Figure 16: Comparison of the experimental and simulated FRF of the fully bolted frame.

- $\delta\left[Y_{L 1}(\theta)\right]=\delta\left[Y_{L 4}(\theta)\right]=0.09$

- $\delta\left[Y_{L 2}(\theta)\right]=\delta\left[Y_{L 3}(\theta)\right]=0.22$.

The average FRF and the envelope FRF obtained with 1000 samples of the input random stiffnesses have been computed with SMR1 and SMR2 and are presented on Figure 16. As for the case of the frame with two bolts, it can be noticed that results obtained with SMR1 and SMR2 are slightly similar, which illustrates that SMR1 is again sufficient to characterize the vibrational behaviour of this frame.

It can be noticed that the simulated vibrational behaviour of the fully bolted frame does not properly fit the experimental FRF. Moreover this assessment must be considered in the light of the simplicity of the model used for modelling the whole structure especially the joints. Overall, the variability of the vibrational behaviour of this structure is well predicted, nevertheless, the simulation results show that averaged eigenfrequencies are slightly over-estimated for the highest eigenfrequency. In addition, these frequency shifts could be partly explained by the fact that this frame is statically indeterminate. Van Buren et al. [29] have observed experimentally that the tightening sequence of a bolted assembly has a significant influence on the structure eigenfrequency. More specifically, the bolt tightening sequence induces some additional stress in joint's interfaces. Consequently, the initial stress state of the joints of the fully bolted frame could differ from the initial state of the joint used for the identification procedure. This phenomenon is probably accentuated by the aluminium-aluminium contact characteristics which facilitate the stick of the joint interfaces. As a consequence, the stick of the joint interface stiffens the joints and tends to increase the eigenfrequencies.

\section{Conclusion}

This paper presents a straightforward approach to implement numerical tools allowing to robustly design light assembled structures. The proposed strategy is based on a separation of the difficulties and assumes that the uncertainties of the whole problem are located in the interfaces between the parts of the assembly whereas the parts are linear and deterministic. As a first step, a nominal model of joint is selected and identified. In the context of this work, this identification procedure is based on an experimental setup in order to ensure a certain consistency with the actual behaviour of lightweight structures. As a second step, this nominal model is extended to propose a stochastic model of joint by randomizing its parameters according to the maximum entropy principle. Finally, a stochastic model identification procedure is proposed. This identification is based on the non-intrusive approach referred to as the Stochastic Model Reduction (SMR) approach [27]. The commercial software MSC Nastran has been used to obtain the mathematical operators required to built SMR. This procedure, which can be seen as an approximation of the maximum likelihood principle, is defined as an optimisation 
problem and takes advantage of the accuracy and efficiency in terms of computation time of the SMR approach. A representative academic structure inspired by work from the literature [29] has been chosen as a guideline for this work. This structure, corresponding to a frame made of three bolted beams, is used to develop and illustrate each step of the construction of the stochastic model of joint. The last part of this work allows to validate the proposed approach using two configurations of bolted frame. The experimental variability of the vibrational behaviour of these frames is compared to the variability computed with the stochastic model of joint and the SMR approach. It is concluded that, although the "just sufficient" nominal model (on which the stochastic model is based) seems to be sensitive to the bolt tightening procedure, the proposed approach allows to tackle the variability of the actual structure using a pragmatic and straightforward procedure. Despite this approach has been implemented considering the case of a light assembled structures, it can be easily extended to massive 3D assembled structures or to glued structures or structures with multi-material.

At last, one of the current limitations of the proposed approach lies in the identification procedure of the stochastic model of joint presented here. Indeed, it is based on the experimental variability of the structure which could be time consuming to obtained. Moreover, it requires to realize several configurations of the studied structure which could also represent a certain financial cost. A numerical experiment based on a high fidelity numerical model (microscopic scale) of the joint would allow to overcome these drawbacks. This numerical tool would also provide a good framework to investigate on which physical parameters could be behind the limitation of the nominal model of joint used in this present paper. In addition, future works will focus on the consideration of damping in the whole mechanical model and especially in the joint behaviour. Damping could also be considered as a source of uncertainty, this would allow us to improve the stochastic prediction of assembled structures. Since the efficient stochastic model is built and precisely identified, development of efficient robust design strategies for industrial applications in dynamics constitutes an highly interesting outlook.

\section{References}

[1] M. S. Phadke. Quality Engineering Using Robust Design. 1st. Upper Saddle River, NJ, USA: Prentice Hall PTR, 1995 (cit. on p. 2).

[2] C. Zang, M. I. Friswell, and J. E. Mottershead. "A review of robust optimal design and its application in dynamics." In: Computers \&3 Structures 83.4 (2005), pp. 315-326. DOI: 10.1016 /j.compstruc.2004.10.007 (cit. on p. 2).

[3] N. W. Hagood and A. von Flotow. "Damping of structural vibrations with piezoelectric materials and passive electrical networks." In: Journal of Sound and Vibration 146.2 (1991), pp. 243-268. DOI: 10.1016/0022-460X (91)90762-9 (cit. on p. 2).

[4] O. Thomas, J. Ducarne, and J.-F. Deü. "Performance of piezoelectric shunts for vibration reduction." In: Smart Materials and Structures 21.1 (2012), p. 16. DOI: 10.1088/0964-1726/21/1/01 5008 (cit. on p. 2).

[5] B. Lossouarn, M. Aucejo, and J.-F. Deü. "Multimodal coupling of periodic lattices and application to rod vibration damping with a piezoelectric network." In: Smart Materials and Structures 24.4 (2015), p. 13. DOI: 10.1088/0964-1726/24/4/045018 (cit. on p. 2).

[6] J. C. H. Chang and T. T. Soong. "Structural control using active tuned mass dampers." In: Journal of the Engineering Mechanics Division 106.6 (1980), pp. 1091-1098 (cit. on p. 2).

[7] M. Gutierrez Soto and H. Adeli. "Tuned Mass Dampers." In: Archives of Computational Methods in Engineering 20.4 (Dec. 2013), pp. 419-431. DOI: 10.1007/s11831-013-9091-7 (cit. on p. 2).

[8] H.-G. Beyer and B. Sendhoff. "Robust optimization - A comprehensive survey." In: Computer Methods in Applied Mechanics and Engineering 196.33 (2007), pp. 3190-3218. DOI: 10.1016 /j.cma.2007.03.003 (cit. on p. 2). 
Martin Ghienne, Luc Laurent \& Claude Blanzé. Robust characterization of the vibrational behaviour of light assembled structures with random parameters. Mechanical Systems and Signal Processing, 136, 2020. doi: 10.1016/j.ymssp.2019.106510

[9] B. D. Youn, K. K. Choi, and L. Du. "Enriched Performance Measure Approach for ReliabilityBased Design Optimization." In: AIAA Journal 43.4 (2005), pp. 874-884. DOI: 10.2514/1.6648 (cit. on p. 2).

[10] C. Soize. "Stochastic modeling of uncertainties in computational structural dynamics - Recent theoretical advances." In: Journal of Sound and Vibration 332.10 (2013), pp. 2379-2395. DOI: 10.1016/j.jsv.2011.10.010 (cit. on pp. 2, 12, 15).

[11] A. Batou, C. Soize, and S. Audebert. "Model identification in computational stochastic dynamics using experimental modal data." In: Mechanical Systems and Signal Processing 50-51 (2015), pp. 307-322. DOI: 10.1016/j.ymssp.2014.05.010 (cit. on pp. 2, 12).

[12] C. Blanzé and L. Champaney. "A computational strategy for the random response of assemblies of structures." In: International Journal of Solids and Structures 41.22-23 (2004), pp. 6383-6405. DOI: $10.1016 /$ j.ijsolstr.2004.05.003 (cit. on pp. 2, 12).

[13] S. Audebert, I. Zentner, and A. Mikchevitch. "Variability and propagation of uncertainties on modal simulations of a built-up structure (SICODYN benchmark)." In: Proceedings of the 8th International Conference on Structural Dynamics - EURODYN 2011. Ed. by G. de Roeck et al. Leuwen, Belgium, Apr. 2011, pp. 3000-3007 (cit. on p. 3).

[14] S. Audebert, A. Mikchevitch, and I. Zentner. "SICODYN international benchmark on dynamic analysis of structure assemblies: variability and numerical-experimental correlation on an industrial pump (part 2)." In: Mechanics \& Industry 15.1 (2014), pp. 1-17. DOI: 10.1051/meca/2014 010 (cit. on p. 3).

[15] R. Ghanem et al. "Modal properties of a space-frame with localized system uncertainties." In: 8th ASCE Specialty Conference of Probabilistic Mechanics and Structural Reliability, ASCE. 2000 (cit. on p. 3).

[16] M. P. Mignolet, P. Song, and X. Q. Wang. "A stochastic Iwan-type model for joint behavior variability modeling." In: Journal of Sound and Vibration 349 (2015), pp. 289-298. DOI: 10.101 6/j.jsv.2015.03.032 (cit. on pp. 3, 15-16).

[17] W. D. Iwan. "A Distributed-Element Model for Hysteresis and Its Steady-State Dynamic Response." In: Journal of Applied Mechanics 33.4 (Dec. 1966), pp. 893-900. DOI: 10.1115/1.3625 199 (cit. on pp. 3, 5-6).

[18] W. D. Iwan. "On a Class of Models for the Yielding Behavior of Continuous and Composite Systems." In: Journal of Applied Mechanics 34.3 (Sept. 1967), pp. 612-617. DOI: 10.1115/1.360 7751 (cit. on pp. 3, 5-6).

[19] S. Daouk et al. "Uncertainties in structural dynamics: overview and comparative analysis of methods." In: Mechanics \& Industry 16.4 (2015), p. 404. DOI: $10.1051 / \mathrm{meca} / 2015010$ (cit. on pp. 3, 12).

[20] G. S. Fishman. Monte Carlo - Concepts, Algorithms, and Applications. Springer New York, 1996. DOI: $10.1007 / 978-1-4757-2553-7$ (cit. on p. 3).

[21] M. Kleiber and T. D. Hien. "The stochastic finite element method (basic perturbation technique and computer implementation)." In: Applied Stochastic Models and Data Analysis 10.4 (1992), pp. 297-297. DOI: 10.1002/asm.3150100412 (cit. on p. 3).

[22] E. Balmés. "Efficient sensitivity analysis based on finite element model reduction." In: Proc. IMAC XVII, SEM. Santa Barbara, CA, 1998, pp. 1168-1174 (cit. on p. 3).

[23] P. Ladevèze, G. Puel, and T. Romeuf. "Lack of knowledge in structural model validation." In: Computer Methods in Applied Mechanics and Engineering 195.37 (2006). John H. Argyris Memorial Issue. Part I, pp. 4697-4710. DOI: 10.1016/j.cma.2005.10.017 (cit. on pp. 3, 12).

[24] W. Gao. "Interval natural frequency and mode shape analysis for truss structures with interval parameters." In: Finite Elements in Analysis and Design 42.6 (2006), pp. 471-477. DOI: https: //doi.org/10.1016/j.finel.2005.09.003 (cit. on p. 3). 
Martin Ghienne, Luc Laurent \& Claude Blanzé. Robust characterization of the vibrational behaviour of light assembled structures with random parameters. Mechanical Systems and Signal Processing,

136, 2020. doi: 10.1016/j.ymssp.2019.106510

[25] W. Gao. "Natural frequency and mode shape analysis of structures with uncertainty." In: $M e-$ chanical Systems and Signal Processing 21.1 (2007), pp. 24-39. DOI: https://doi.org/10.1016 /j.ymssp.2006.05.007 (cit. on p. 3).

[26] D. Moens and D. Vandepitte. "Interval sensitivity theory and its application to frequency response envelope analysis of uncertain structures." In: Computer Methods in Applied Mechanics and Engineering 196.21 (2007), pp. 2486-2496. DOI: https://doi.org/10.1016/j.cma.2007.01.00 6 (cit. on p. 3).

[27] M. Ghienne, C. Blanzé, and L. Laurent. "Stochastic model reduction for robust dynamical characterization of structures with random parameters." In: Comptes Rendus Mécanique 345.12 (2017), pp. 844-867. DOI: 10.1016/j.crme.2017.09.006 (cit. on pp. 4, 13-14, 22).

[28] M. Ghienne. "Conception et caractérisation de liaisons boulonnées pour la réduction robuste de vibrations de structures." PhD thesis. Paris: Conservatoire national des arts et métiers - CNAM, Dec. 2017 (cit. on pp. 4, 8, 13-14, 16).

[29] K. L. Van Buren et al. "A case study to quantify prediction bounds caused by model-form uncertainty of a portal frame." In: Mechanical Systems and Signal Processing 50-51 (2015), pp. 1126. DOI: $10.1016 / j$.ymssp. 2014.05 .001 (cit. on pp. 4-5, 7, 19, 22-23).

[30] Y. Song et al. "Simulation of dynamics of beam structures with bolted joints using adjusted Iwan beam elements." In: Journal of Sound and Vibration 273.1-2 (2004), pp. 249-276. DOI: 10.1016/S0022-460X (03)00499-1 (cit. on pp. 5-7).

[31] L. Gaul, J. Lenz, and D. Sachau. "Active Damping of Space Structures by Contact Pressure Control in Joints." In: Mechanics of Structures and Machines 26.1 (1998), pp. 81-100. DOI: 10.1080/08905459808945421 (cit. on pp. 5-6).

[32] J. Esteban and C. A. Rogers. "Energy dissipation through joints: theory and experiments." In: Computers \& Structures 75.4 (2000), pp. 347-359. DOI: 10 . 1016/S0045-7949(99) 00096-6 (cit. on pp. 5-6).

[33] U. Lee. "Dynamic Characterization of the Joints in a Beam Structure by Using Spectral Element Method." In: Shock and Vibration 8.6 (2001). DOI: 10.1155/2001/254020 (cit. on pp. 5-6).

[34] S. Bograd et al. "Modeling the dynamics of mechanical joints." In: Mechanical Systems and Signal Processing 25.8 (2011), pp. 2801-2826. DOI: 10.1016/j.ymssp. 2011.01 .010 (cit. on pp. 5, 7).

[35] M. H. Mayer and L. Gaul. "Segment-to-segment contact elements for modelling joint interfaces in finite element analysis." In: Mechanical Systems and Signal Processing 21.2 (2007), pp. 724-734. DOI: $10.1016 / j$.ymssp. 2005.10.006 (cit. on pp. 5, 7).

[36] H. Ahmadian and H. Jalali. "Generic element formulation for modelling bolted lap joints." In: Mechanical Systems and Signal Processing 21.5 (2007), pp. 2318-2334. DOI: 10.1016/j .ymssp. 2 006.10 .006 (cit. on pp. 5, 7, 10).

[37] H. Ahmadian and H. Jalali. "Identification of bolted lap joints parameters in assembled structures." In: Mechanical Systems and Signal Processing 21.2 (2007), pp. 1041-1050. DOI: 10.1016 /j.ymssp.2005.08.015 (cit. on pp. 5, 7).

[38] L. Gaul and R. Nitsche. "The Role of Friction in Mechanical Joints." In: Applied Mechanics Reviews 54.2 (Mar. 2001), pp. 93-106. DOI: 10.1115/1.3097294 (cit. on pp. 5-6).

[39] R. A. Ibrahim and C. L. Pettit. "Uncertainties and dynamic problems of bolted joints and other fasteners." In: Journal of Sound and Vibration 279.3 - 5 (2005), pp. 857-936. DOI: 10.1016 $/$ j.jsv.2003.11.064 (cit. on pp. 5-6).

[40] N. Peyret. "Dissipation de l'énergie mécanique dans les assemblages : effet du frottement en sollicitation dynamique." PhD thesis. Université Paris-Est, 2012 (cit. on pp. 5-6).

[41] H. Festjens, G. Chevallier, and J. Dion. "Nonlinear model order reduction of jointed structures for dynamic analysis." In: Journal of Sound and Vibration 333.7 (2014), pp. 2100-2113. DOI: 10.1016/j.jsv.2013.11.039 (cit. on pp. 5-6). 
Martin Ghienne, Luc Laurent \& Claude Blanzé. Robust characterization of the vibrational behaviour of light assembled structures with random parameters. Mechanical Systems and Signal Processing, 136, 2020. doi: 10.1016/j.ymssp.2019.106510

[42] P. R. Dahl. A solid friction model. Tech. rep. TOR-158(3107-18). El segundo, CA: The Aerospace Corporation, 1968 (cit. on pp. 5-6).

[43] C. Canudas-De-Wit et al. "A new model for control of systems with friction." In: IEEE Transactions on Automatic Control 40.3 (Mar. 1995), pp. 419-425. DOI: 10.1109/9.376053 (cit. on pp. 5-6).

[44] K. C. Valanis. "A friction viscoplasticity without a yield surface." In: Archive Mech 23.4 (1970), pp. 517-551 (cit. on pp. 5-6).

[45] R. Bouc. "Forced vibration of mechanical systems with hysteresis." In: Proceedings of the Fourth Conference on Nonlinear Oscillations. Prague, 1967, pp. 32-39 (cit. on pp. 5-6).

[46] Y. K. Wen. "Method of random vibration of hysteretic systems." In: Journal of the Engineering Mechanics Division 102.2 (1976), pp. 249-263 (cit. on pp. 5-6).

[47] G. Masing. "Eigenspannungen und Verfestigung beim Messing." In: Proceedings of the 2nd International Congress of Applied Mechanics. 1926, pp. 332-335 (cit. on pp. 5-6).

[48] D. J. Segalman and M. J. Starr. Relationships among certain joint constitutive models. Tech. rep. SAND2004-4321. Albuquerque, New Mexico: Sandia National Laboratories, 2004. DOI: 10.2172 /919196 (cit. on pp. 5-6).

[49] D. J. Segalman. "A Four-Parameter Iwan Model for Lap-Type Joints." In: Journal of Applied Mechanics 72.5 (Feb. 2005), pp. 752-760. DOI: 10.1115/1.1989354 (cit. on pp. 5-6).

[50] L. E. Goodman and J. H. Klumpp. "Analysis of slip damping with reference to turbine blade vibration." In: Journal of Applied Mechanics 23 (1956), p. 421 (cit. on pp. 5-6).

[51] A. F. Metherell and S. V. Diller. "Instantaneous Energy Dissipation Rate in a Lap Joint-Uniform Clamping Pressure." In: Journal of Applied Mechanics 35.1 (Mar. 1968), pp. 123-128. DOI: 10.1 115/1.3601124 (cit. on pp. 5-6).

[52] Y. Song et al. "Effect of Pressure Distribution on Energy Dissipation in a Mechanical Lap Joint." In: AIAA Journal 43.2 (Feb. 2005), pp. 420-425. DOI: 10.2514/1.10139 (cit. on pp. 5-6).

[53] A. D. Crocombe et al. "Estimating the energy dissipated in a bolted spacecraft at resonance." In: Computers \& Structures 84.5-6 (2006), pp. 340-350. DOI: 10.1016/ j . compstruc. 2005.09.024 (cit. on pp. 5-6).

[54] M. Oldfield, H. Ouyang, and J. E. Mottershead. "Simplified models of bolted joints under harmonic loading." In: Computers \& Structures 84.1-2 (2005), pp. 25-33. DOI: 10.1016/j . compstruc . 2005.09.007 (cit. on pp. 5-6).

[55] R. D. Mindlin. "Compliance of elastic bodies in contact." In: Journal of Applied Mechanics 16 (1949), pp. 259-268 (cit. on pp. 5-6).

[56] N. Peyret, G. Chevallier, and J.-L. Dion. "Dynamic Damping in Joints: Multiscale Model Taking into Account Defects in a Nominally Plane Surface." In: International Journal of Applied Mechanics 8.8 (2016), p. 1650097. DOI: 10.1142/S1758825116500976 (cit. on pp. 5-6).

[57] L. Kogut and I. Etsion. "A Static Friction Model for Elastic-Plastic Contacting Rough Surfaces." In: Journal of Tribology 126.1 (Jan. 2004), pp. 34-40. DOI: 10.1115/1.1609488 (cit. on pp. 5-6).

[58] F. Gant, L. Champaney, and P. Rouch. "Modeling of the bolted joint behavior variability with the Lack of Knowledge theory." In: ICCES 2010 - International Conference on Computational and Experimental Engineering and Sciences. 2010 (cit. on pp. 7, 12).

[59] N. Peyret et al. "Micro-slip induced damping in planar contact under constant and uniform normal stress." In: International Journal of Applied Mechanics 02.02 (2010), pp. 281-304. DOI: 10.1142/S1758825110000597 (cit. on p. 10).

[60] L. Gaul and J. Lenz. "Nonlinear dynamics of structures assembled by bolted joints." In: Acta Mechanica 125.1 (1997), pp. 169-181. DOI: 10.1007/BF01177306 (cit. on p. 10). 
Martin Ghienne, Luc Laurent \& Claude Blanzé. Robust characterization of the vibrational behaviour of light assembled structures with random parameters. Mechanical Systems and Signal Processing, 136, 2020. doi: 10.1016/j.ymssp.2019.106510

[61] D. J. Ewins. Modal testing - Theory and practice. Vol. ch 3, pp. 158-168. Mechanical engineering research studies, 1984 (cit. on p. 10).

[62] L. A. Zadeh. "Fuzzy sets as a basis for a theory of possibility." In: Fuzzy Sets and Systems 100 (1999), pp. 9-34. DOI: 10.1016/S0165-0114(99)80004-9 (cit. on p. 12).

[63] C. Soize. "Generalized probabilistic approach of uncertainties in computational dynamics using random matrices and polynomial chaos decompositions." In: International Journal for Numerical Methods in Engineering 81.8 (2010), pp. 939-970. DOI: 10.1002/nme.2712 (cit. on p. 12).

[64] C. Blanzé and P. Rouch. "Analysis of structures with stochastic interfaces in the mediumfrequency range." In: Journal of Computational Acoustics 13.04 (2005), pp. 711-729. DOI: 10 .1142/S0218396X05002876 (cit. on p. 12).

[65] M. Shinozuka and C. J. Astill. "Random Eigenvalue Problems in Structural Analysis." In: $A I A A$ Journal 10.4 (Apr. 1972), pp. 456-462. DOI: 10.2514/3.50119 (cit. on p. 12).

[66] R. Ghanem and P. D. Spanos. "Polynomial Chaos in Stochastic Finite Elements." In: Journal of Applied Mechanics 57.1 (Mar. 1990), pp. 197-202. DOI: 10.1115/1.2888303 (cit. on p. 12).

[67] M. Papadrakakis and A. Kotsopulos. "Parallel solution methods for stochastic finite element analysis using Monte Carlo simulation." In: Computer Methods in Applied Mechanics and Engineering 168.1 (1999), pp. 305-320. DOI: 10.1016/S0045-7825(98)00147-9 (cit. on p. 12).

[68] B. Van den Nieuwenhof and J.-P. Coyette. "Modal approaches for the stochastic finite element analysis of structures with material and geometric uncertainties." In: Computer Methods in Applied Mechanics and Engineering 192.33 (2003), pp. 3705-3729. DOI: 10.1016/S0045-7825(03) 00371 -2 (cit. on p. 12).

[69] B. Sudret and A. Der Kiureghian. Stochastic Finite Element Methods and Reliability: A Stateof-the-Art Report. Tech. rep. UCB/SEMM-2000/08. Department of Civil \& Environmental Engineering, University of California, Berkeley, Nov. 2000 (cit. on pp. 12-13).

[70] G. Stefanou. "The stochastic finite element method: Past, present and future." In: Computer Methods in Applied Mechanics and Engineering 198.9-12 (2009), pp. 1031-1051. DOI: 10.1016 /j.cma.2008.11.007 (cit. on p. 12).

[71] J. D. Collins and W. T. Thomson. "The eigenvalue problem for structural systems with statistical properties." In: AIAA Journal 7.4 (Apr. 1969), pp. 642-648. DOI: 10.2514/3.5180 (cit. on p. 12).

[72] S. Adhikari and M. I. Friswell. "Random matrix eigenvalue problems in structural dynamics." In: International Journal for Numerical Methods in Engineering 69.3 (2007), pp. 562-591. DOI: 10.1002/nme.1781 (cit. on p. 12).

[73] N. Wiener. "The Homogeneous Chaos." In: American Journal of Mathematics 60.4 (Oct. 1938), pp. 897-936. DOI: 10.2307/2371268 (cit. on p. 12).

[74] L. Hinke. "Modelling approaches for the low-frequency analysis of built-up structures with nondeterministic properties." PhD thesis. University of Southampton, Apr. 2008 (cit. on p. 13).

[75] L. Hinke et al. "Component mode synthesis as a framework for uncertainty analysis." In: Journal of Sound and Vibration 324.1 (2009), pp. 161-178. DOI: 10.1016/j.jsv.2009.01.056 (cit. on p. 13).

[76] S. Audebert and F. Fall-Lo. "Uncertainty analysis on a pump assembly using component mode synthesis." In: 4th ECCOMAS Thematic Conference on Computational Methods in Structural Dynamics and Earthquake Engineering - COMPDYN 2013. Ed. by M. Papadrakakis, V. Papadopoulos, and V. Plevris. Dec. 2013 (cit. on p. 13).

[77] R. Ghanem and D. Ghosh. "Efficient characterization of the random eigenvalue problem in a polynomial chaos decomposition." In: International Journal for Numerical Methods in Engineering 72.4 (2007), pp. 486-504. DOI: 10.1002/nme.2025 (cit. on p. 13). 
[78] G. Blatman and B. Sudret. "Adaptive sparse polynomial chaos expansion based on least angle regression." In: Journal of Computational Physics 230.6 (2011), pp. 2345-2367. DOI: 10.1016 /j.jcp.2010.12.021 (cit. on p. 13).

[79] C. Soize. Stochastic Models of Uncertainties in Computational Mechanics. American Society of Civil Engineers, 2012. DOI: 10.1061/9780784412237 (cit. on p. 15).

[80] E. T. Jaynes. "Information theory and statistical mechanics." In: Phys. Rev. 106 (4 May 1957), pp. 620-630. DOI: 10.1103/PhysRev.106.620 (cit. on p. 15).

[81] S. Daouk. "Propagation d'incertitudes à travers des modèles dynamiques d'assemblages de structures mécaniques." PhD thesis. Université Paris-Saclay, Nov. 2016 (cit. on p. 19). 